\title{
Enumerating partial Latin rectangles
}

\author{
Raúl M. Falcón* \\ Department of Applied Mathematics I \\ University of Seville, Spain. \\ rafalgan@us.es \\ Rebecca J. Stones ${ }^{\dagger}$ \\ College of Computer Science \\ Nankai University, Tianjin, China \\ School of Mathematical Sciences and Faculty of Information Technology \\ Monash University, Australia. \\ Department of Mathematics and Statistics \\ Dalhousie University, Halifax, Canada. \\ rebecca.stones82@gmail.com
}

Submitted: Oct 26, 2019; Accepted: May 19, 2020; Published: Jun 12, 2020

(C) Raúl M. Falcón and Rebecca J. Stones. Released under the CC BY-ND license (International 4.0).

\begin{abstract}
This paper deals with different computational methods to enumerate the set $\operatorname{PLR}(r, s, n ; m)$ of $r \times s$ partial Latin rectangles on $n$ symbols with $m$ non-empty cells. For fixed $r, s$, and $n$, we prove that the size of this set is given by a symmetric polynomial of degree $3 \mathrm{~m}$, and we determine the leading terms (the monomials of degree $3 m$ through $3 m-9$ ) using inclusion-exclusion. For $m \leqslant 13$, exact formulas for these symmetric polynomials are determined using a chromatic polynomial method. Adapting Sade's method for enumerating Latin squares, we compute the exact size of $\operatorname{PLR}(r, s, n ; m)$, for all $r \leqslant s \leqslant n \leqslant 7$, and all $r \leqslant s \leqslant 6$ when $n=8$. Using an algebraic geometry method together with Burnside's Lemma, we enumerate isomorphism, isotopism, and main classes when $r \leqslant s \leqslant n \leqslant 6$. Numerical results have been cross-checked where possible.
\end{abstract}

Mathematics Subject Classifications: 05B15

Keywords: Partial Latin rectangle, isomorphism, isotopism, main class, inclusionexclusion, chromatic polynomial, algebraic geometry.

\footnotetext{
*Supported by the research project FQM-016 from Junta de Andalucía.

†Supported by her NSFC Research Fellowship for International Young Scientists (grant numbers: 11450110409, 11550110491), NSF China grant 61170301, and the Thousand Youth Talents Plan in Tianjin.
} 


\section{Introduction}

Let $[n]:=\{1,2, \ldots, n\}$. An $r \times s$ partial Latin rectangle $L=\left(l_{i j}\right)$ on the symbol set $[n] \cup\{\cdot\}$ is an $r \times s$ matrix such that each row and each column has at most one copy of any symbol in $[n]$. Here, $r, s$, and $n$ are arbitrary positive integers, and we admit the possibility that $n<\min \{r, s\}$. If $r=s=n$, then this constitutes a partial Latin square of order $n$. The cells containing the symbol - are considered empty, and we say that $l_{i j}$ is undefined. An entry of $L$ is any triple $\left(i, j, l_{i j}\right) \in[r] \times[s] \times[n]$. The set of all entries of $L$ is called its entry set, which is denoted $E(L)$. The weight of $L$ is its number of non-empty cells, that is, the size of its entry set. Let $\operatorname{PLR}(r, s, n ; m)$ denote the set of $r \times s$ partial Latin rectangles on the symbol set $[n] \cup\{\cdot\}$ of weight $m$ and let $\operatorname{PLR}(r, s, n)=\cup_{0 \leqslant m \leqslant r s} \operatorname{PLR}(r, s, n ; m)$. Let $\operatorname{PLS}(n ; m)=\operatorname{PLR}(n, n, n ; m)$ be the set of partial Latin squares of weight $m$. For $m=n^{2}$, this is the set of Latin squares of order $n$.

For each positive integer $t \in \mathbb{Z}^{+}$, let $S_{t}$ denote the symmetric group on the set $[t]$.

- The isotopism group $\mathfrak{I}_{r, s, n}:=S_{r} \times S_{s} \times S_{n}$ acts on the set $\operatorname{PLR}(r, s, n ; m)$, with the isotopism $\Theta=(\alpha, \beta, \gamma)$ permuting the rows according to $\alpha$, the columns according to $\beta$, and the symbols according to $\gamma$. This gives the isotopic partial Latin rectangle $L^{\Theta} \in$ $\operatorname{PLR}(r, s, n ; m)$, whose entry set is $E\left(L^{\Theta}\right)=\left\{\left(\alpha(i), \beta(j), \gamma\left(l_{i, j}\right)\right):\left(i, j, l_{i j}\right) \in E(L)\right\}$.

- The symmetric group $S_{n}$ is isomorphic to the subgroup $\left\{(\alpha, \alpha, \alpha): \alpha \in S_{n}\right\}$ of the isotopism group $\mathfrak{I}_{n, n, n}$ via the isomorphism $\alpha \mapsto(\alpha, \alpha, \alpha)$. In this regard, the isomorphism group $S_{n}$ acts on the set $\operatorname{PLS}(n ; m)$, with $\alpha \in S_{n}$ mapping $L$ to $L^{(\alpha, \alpha, \alpha)}$.

- Let $\pi \in S_{3}$ and $L \in \operatorname{PLR}\left(d_{1}, d_{2}, d_{3} ; m\right)$. The parastrophic partial Latin rectangle $L^{\pi} \in \operatorname{PLR}\left(d_{\pi(1)}, d_{\pi(2)}, d_{\pi(3)} ; m\right)$ is defined so that its entry set is $E\left(L^{\pi}\right)=$ $\left\{\left(p_{\pi(1)}, p_{\pi(2)}, p_{\pi(3)}\right):\left(p_{1}, p_{2}, p_{3}\right) \in E(L)\right\}$. The permutation $\pi$ is said to be a parastrophism. Since parastrophisms may not preserve the dimensions of partial Latin rectangles, the parastrophism group $S_{r, s, n}$ is defined as the stabilizer of the ordered triple $(r, s, n)$ under the action $\left(d_{1}, d_{2}, d_{3}\right) \stackrel{\pi}{\mapsto}\left(d_{\pi(1)}, d_{\pi(2)}, d_{\pi(3)}\right)$ by $S_{3}$.

- The paratopism group $\mathfrak{P}_{r, s, n}:=\mathfrak{I}_{r, s, n} \rtimes S_{r, s, n}$ acts on the set $\operatorname{PLR}(r, s, n ; m)$ so that each paratopism $(\Theta, \pi)$ maps $L$ to the paratopic partial Latin rectangle $L^{(\Theta, \pi)}=$ $\left(L^{\pi}\right)^{\Theta}$. When $\pi=$ Id (i.e., the trivial permutation in $S_{3}$ ) the isotopism group arises as a normal subgroup of the paratopism group.

Orbits of $\operatorname{PLR}(r, s, n ; m)$ under the isotopism, isomorphism, and paratopism groups are equivalence classes, called isotopism, isomorphism, and main classes (sometimes known as species), respectively. The stabilizer subgroups under these groups are called autotopism, automorphism, and autoparatopism groups, respectively. Let $\operatorname{PLR}((\Theta, \pi))$ and $\operatorname{PLR}((\Theta, \pi) ; m)$ denote, respectively, the subsets of partial Latin rectangles in the sets $\operatorname{PLR}(r, s, n)$ and $\operatorname{PLR}(r, s, n ; m)$ that admit an autoparatopism $(\Theta, \pi) \in \mathfrak{P}_{r, s, n}$.

The goal of this paper is to find methods for computing the size of $\operatorname{PLR}(r, s, n ; m)$, along with its equivalence class sizes. It is unrealistic to expect a succinct solution to both problems for arbitrary $r, s, n$, and $m$, since they include in particular the number of Latin 
squares of given order $n$, which is a long-standing research problem in combinatorics. This is known only for order $n \leqslant 11[41,53]$; see $[61,62,70]$ for some related results on Latin rectangles. Currently, the number of partial Latin rectangles is known only for $r, s, n \leqslant 6$ $[27,28,30]$. In the present paper, we extend previous results by enumerating (a) fixedweight partial Latin rectangles, (b) partial Latin rectangles for small $m$, and (c) partial Latin rectangles for small $r, s$, and $n$.

The number of isotopism, isomorphism, and main classes of Latin squares has been determined [41, 50] for order $n \leqslant 11$, whereas for partial Latin rectangles, these numbers were computed [34] for $r, s, n \leqslant 6$. Adams, Bean, and Khodkar [1] enumerated main classes of partial Latin squares of order $n \leqslant 6$ that constitute critical sets. More recently, the number of main classes of partial Latin rectangles with at most 12 entries was calculated [20, 73]. See also [31] for a recent survey on the theory of isotopisms.

Autoparatopisms and symmetry for partial Latin squares were studied in $[63,3]$ and several constructions of partial Latin rectangles with trivial autotopism groups for various autoparatopism groups was given in [36]. Computational methods for determining autotopism groups of partial Latin rectangles were compared in [17, 18, 69, 29]. For Latin squares of order $n \leqslant 17$, identifying when $\# \operatorname{PLR}((\Theta, \pi)) \neq 0$ (throughout this paper \# denotes the cardinality of a set) was done for isotopisms in [64] and paratopisms in [56], with prior work in $[26,32]$.

Symmetries of Latin squares and rectangles have been studied in a wide range of contexts, e.g., enumeration [57, 65, 66], subsquares [13, 55], the Alon-Tarsi Conjecture [21, 67], quasigroups and loops [8, 44, 45, 54], special kinds of symmetries [15, 33, 42, 72], and in their own right $[9,14,24,25]$. They are beginning to find applications in secret sharing schemes $[23,71,75]$, erasure codes [76, 68], and graph coloring games [5, 4].

The remainder of the paper is organized as follows. The three following sections deal with different combinatorial methods that enable us to determine the size of the set $\operatorname{PLR}(r, s, n ; m)$. Thus, we show in Section 2 an inclusion-exclusion method that demonstrates \#PLR $(r, s, n ; m)$ for fixed $m$ is given by a symmetric polynomial of degree $3 m$. In Section 3, we describe a chromatic polynomial method that gives exact formulas for this symmetric polynomial, which we compute for $m \leqslant 13$. Finally, we show in Section 4 an adaptation of Sade's method (which efficiently enumerates Latin squares) to partial Latin rectangles, which enables us to determine explicitly the number $\# \operatorname{PLR}(r, s, n ; m)$ for all $r \leqslant s \leqslant n \leqslant 7$, and all $r \leqslant s \leqslant 6$ when $n=8$. Section 5 describes an algebraic method for computing \#PLR $((\Theta, \pi) ; m)$ and also the number of isotopisms between two given partial Latin rectangles. Further, in Section 6 we use the Orbit-Stabilizer Theorem and Burnside's Lemma to compute the size of isomorphism, isotopism and main classes. Section 7 describes the computational results and the implementations of the various methods. In Section 8 we comment on these computational results have been cross-checked in order to ensure their accuracy. A glossary of the most common symbols that are used throughout the paper is shown in Appendix A. To improve the readability of the paper, tables are in Appendix B. 


\section{Inclusion-exclusion method}

In this section, we describe an inclusion-exclusion method (based in turn on a graph theoretic approach) for finding formulas for the size of $\operatorname{PLR}(r, s, n ; m)$, with $m \geqslant 1$. To this end, we modify conveniently the method for enumerating partial orthomorphisms of finite cyclic groups given in [66]. At first glance, this may seem surprising as partial Latin rectangles and partial orthomorphisms are largely unrelated (unless we impose some symmetry, which we don't in the context of this section). The similarity between these two types of objects is that both partial Latin rectangles of weight $m$ and partial orthomorphisms with domain size $m$ are equivalent to $m$-sets of ordered triples satisfying certain conditions. Their difference is, precisely, the description of such conditions.

\subsection{Generalized ordered partial Latin rectangles}

Let $\mathcal{S}_{m}=\mathcal{S}(r, s, n ; m)$ be the set of sequences $\mathbf{e}=\left(e_{i}\right)_{i=1}^{m}$, where each $e_{i}=\left(e_{i}[1], e_{i}[2]\right.$, $\left.e_{i}[3]\right)$ is a 3 -tuple in $[r] \times[s] \times[n]$. From any $\mathbf{e} \in \mathcal{S}_{m}$, we construct an $r \times s$ matrix $M=M(\mathbf{e})$ as follows:

- We begin with each cell in $M$ containing the empty multiset $\varnothing$.

- For $i \in[m]$, we add symbol $e_{i}[3]$ in the multiset in cell $\left(e_{i}[1], e_{i}[2]\right)$.

For example, if $r=s=n=m=3$ and $\mathbf{e}=((1,1,1),(1,2,3),(1,1,1))$, then

$$
M(\mathbf{e})=\begin{array}{ccc}
\{1,1\} & \{3\} & \varnothing \\
\varnothing & \varnothing & \varnothing \\
\varnothing & \varnothing & \varnothing
\end{array}
$$

If it turns out that every non-empty multiset in $M$ has cardinality 1 and there are no repeated elements in any row or column of $M$, then $M$ is essentially a partial Latin rectangle (formally, we need to map $\varnothing \mapsto \cdot$ and $\{i\} \mapsto i$ ). For example, if $r=s=n=$ $m=3$ and $\mathbf{e}=((1,1,1),(1,2,3),(2,2,2))$, then

$$
M(\mathbf{e})=\begin{array}{ccc}
\{1\} & \{3\} & \varnothing \\
\varnothing & \{2\} & \varnothing \\
\varnothing & \varnothing & \varnothing
\end{array} \longleftrightarrow \begin{array}{ccc|}
1 & 3 & \cdot \\
\cdot & 2 & \cdot \\
\cdot & \cdot & .
\end{array}
$$

Thus, sequences in $\mathcal{S}_{m}$ are generalized partial Latin rectangles consisting of $m$ ordered entries.

Let $\mathcal{A}_{m}$ be the subset of $\mathcal{S}_{m}$ that gives rise to partial Latin rectangles. Hence,

$$
\left|\mathcal{A}_{m}\right|=m ! \# \operatorname{PLR}(r, s, n ; m)
$$

because we can order the entries in a partial Latin rectangle in $m$ ! ways. For fixed $m$, we define

$$
f_{m}(r, s, n):=\left|\mathcal{A}_{m}\right|
$$


To find a formula for $f_{m}(r, s, n)$, we use inclusion-exclusion on the number of clashes or triples in

$$
C_{m}:=\{[i, j, k]: 1 \leqslant i<j \leqslant m \text { and } k \in\{1,2,3\}\},
$$

which we use to index the possible clashes in $\mathbf{e} \in \mathcal{S}_{m}$ as follows:

Clash $[i, j, 1]$ : When $e_{i}[2]=e_{j}[2]$ and $e_{i}[3]=e_{j}[3]$. This would result in two copies of the same symbol in the same column in $M$ (not necessarily in distinct cells).

Clash $[i, j, 2]$ : When $e_{i}[1]=e_{j}[1]$ and $e_{i}[3]=e_{j}[3]$. This would result in two copies of the same symbol in the same row in $M$ (not necessarily in distinct cells).

Clash $[i, j, 3]$ : When $e_{i}[1]=e_{j}[1]$ and $e_{i}[2]=e_{j}[2]$. This would result in two (not necessarily distinct) symbols in the same cell in $M$.

Any $\mathbf{e} \in \mathcal{S}_{m}$ has a corresponding set of clashes $C_{\mathbf{e}} \subseteq C_{m}$. For any $U \subseteq C_{m}$, define

$$
\mathcal{B}_{U}:=\left\{\mathbf{e} \in \mathcal{S}_{m}: U \subseteq C_{\mathbf{e}}\right\}
$$

i.e., the sequences in $\mathcal{S}_{m}$ that have the clashes in $U$ (and possibly more clashes), and

$$
\mathcal{D}_{U}:=\left\{\mathbf{e} \in \mathcal{S}_{m}: U=C_{\mathbf{e}}\right\}
$$

i.e., the sequences in $\mathcal{S}_{m}$ that have precisely those clashes in $U$ (and no more clashes). By definition,

$$
\mathcal{D}_{U}=\mathcal{B}_{U} \backslash \bigcup_{\substack{V \subseteq C_{m} \\ V \supsetneq U}} \mathcal{B}_{V} .
$$

Hence, by inclusion-exclusion,

$$
\begin{aligned}
\left|\mathcal{D}_{U}\right| & =\left|\mathcal{B}_{U}\right|-\left|\bigcup_{\substack{V \subseteq C_{m} \\
V \supseteq U}} \mathcal{B}_{V}\right| \\
& =\left|\mathcal{B}_{U}\right|+\sum_{\substack{V \subseteq C_{m} \\
V \supsetneq U}}(-1)^{|V|-|U|}\left|\mathcal{B}_{V}\right| \\
& =\sum_{\substack{V \subseteq C_{m} \\
V \supseteq U}}(-1)^{|V|-|U|}\left|\mathcal{B}_{V}\right| .
\end{aligned}
$$

When $U=\varnothing$, we have $\left|\mathcal{D}_{U}\right|=\left|\mathcal{A}_{m}\right|$ and consequently the following lemma.

Lemma 1. For all $m, r, s, n \geqslant 1$, we have

$$
f_{m}(r, s, n)=\sum_{V \subseteq C_{m}}(-1)^{|V|}\left|\mathcal{B}_{V}\right|
$$




\subsection{Graph colorings}

Our next goal is to find an equation for $\left|\mathcal{B}_{V}\right|$ in terms of the number of vertex colorings of an edge-colored graph, satisfying some additional constraints (neither vertex colorings nor edge colorings are required to be proper in the ordinary sense). Given $V \subseteq C_{m}$, we define a graph $G=G(V)$ with an edge coloring $\delta=\delta(V)$ by the following process. We start with the null graph on the vertex set $[m]$, and for each $[i, j, k] \in V$ :

I: If $k=1$, then add a dotted edge between $i$ and $j$.

II: If $k=2$, then add a dashed edge between $i$ and $j$.

III: If $k=3$, then add a dash-dotted edge between $i$ and $j$.

IV: Replace any parallel edges resulting from I-III with a solid edge.

We denote the graph together with its edge coloring generated from $V$ by $(G, \delta)_{V}$. An example of an edge-colored graph generated in this way is given in Figure 1.

\begin{tabular}{|c|}
\multicolumn{1}{c|}{$V$ comprises: } \\
\hline Dotted edges \\
{$[1,2,1]$} \\
{$[1,3,1]$} \\
{$[3,4,1]$} \\
\hline \hline Dashed edges \\
{$[1,3,2]$} \\
{$[1,4,2]$} \\
\hline \hline Dash-dotted edges \\
{$[2,3,3]$} \\
\hline
\end{tabular}

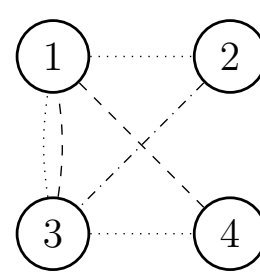

replace parallel edges with solid edges

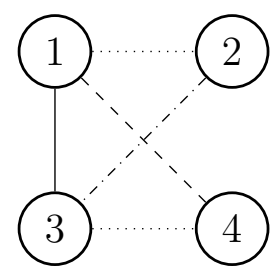

Figure 1: An example of the graph $G=G(V)$, and its edge coloring $\delta=\delta(V)$ (right), for the set of clashes $V \subseteq C_{4}$.

Sequences $\mathbf{e} \in \mathcal{B}_{V}$ are equivalent to a special type of vertex coloring $\phi$ of $(G, \delta)_{V}$, for which we assign to vertex $i \in[m]$ the color

$$
\left(\phi_{1}(i), \phi_{2}(i), \phi_{3}(i)\right):=\left(e_{i}[1], e_{i}[2], e_{i}[3]\right) \in[r] \times[s] \times[n] .
$$

This coloring satisfies the properties:

- If there is a dotted edge between vertices $i$ and $j$, then $\phi_{2}(i)=\phi_{2}(j)$ and $\phi_{3}(i)=$ $\phi_{3}(j)$.

- If there is a dashed edge between vertices $i$ and $j$, then $\phi_{1}(i)=\phi_{1}(j)$ and $\phi_{3}(i)=$ $\phi_{3}(j)$.

- If there is a dash-dotted edge between vertices $i$ and $j$, then $\phi_{1}(i)=\phi_{1}(j)$ and $\phi_{2}(i)=\phi_{2}(j)$. 
- If there is a solid edge between vertices $i$ and $j$, then $\phi_{1}(i)=\phi_{1}(j), \phi_{2}(i)=\phi_{2}(j)$ and $\phi_{3}(i)=\phi_{3}(j)$.

We call such a vertex coloring of $(G, \delta)_{V}$ suitable. Conversely, any suitable vertex coloring of $(G, \delta)_{V}$ with the vertex color set $[r] \times[s] \times[n]$ that satisfies the above four properties is equivalent to a member of $\mathcal{B}_{V}$, thus giving the following lemma.

Lemma 2. For all $V \subseteq C_{m},\left|\mathcal{B}_{V}\right|$ is the number of suitable vertex colorings of $(G, \delta)_{V}$.

We can find a simple formula (Lemma 3) for the number of suitable colorings of $(G, \delta)_{V}$ since each of the three coordinates can be accounted for separately. Let $H_{1}, H_{2}$, and $H_{3}$ respectively be the graphs formed by deleting the dotted, dashed, and dash-dotted edges from $(G, \delta)_{V}$, then ignoring the edge colors. For any graph $H$, let $c(H)$ denote the number of connected components in $H$.

Lemma 3. The number of suitable vertex colorings of $(G, \delta)_{V}$ is

$$
\left|\mathcal{B}_{V}\right|=r^{c\left(H_{1}\right)} s^{c\left(H_{2}\right)} n^{c\left(H_{3}\right)} \text {. }
$$

Proof. In order to be a suitable vertex coloring, the vertices in each component of $H_{1}$ must be assigned colors in $G$ that agree at the first coordinate. We can thus assign the first coordinates of a suitable vertex coloring in $r^{c\left(H_{1}\right)}$ ways. Similar claims hold for $\mathrm{H}_{2}$ and $H_{3}$.

We are now ready to make the following fundamental observation about the polynomials $f_{m}$.

Theorem 4. For fixed $m$, we have that $f_{m}=f_{m}(r, s, n)$ is given by a 3-variable symmetric polynomial with integer coefficients of degree $3 \mathrm{~m}$.

Proof. Lemmas 1 and 3 imply that $f_{m}(r, s, n)=\sum_{V \subseteq C_{m}}(-1)^{|V|}\left|\mathcal{B}_{V}\right|$ where $\left|\mathcal{B}_{V}\right|$ is given by $r^{c\left(H_{1}\right)} s^{c\left(H_{2}\right)} n^{c\left(H_{3}\right)}$ for the graph $(G, \delta)_{V}$. This ensures that $f_{m}(r, s, n)$ is a polynomial in variables $r, s, n$ and has integer coefficients. The leading term is $(r s n)^{m}$, which arises when $V=\varnothing$; for all other $V \subseteq C_{m}$, we see $\left|\mathcal{B}_{V}\right|$ has degree less than $3 m$. Finally, to verify that $f_{m}(r, s, n)$ is a symmetric polynomial, we observe that we can permute the dotted, dashed and dash-dotted styles (or equivalently, permute the third coordinate of the elements in $C_{m}$ ). Each equivalence class under this action contributes

$$
\begin{aligned}
& r^{c\left(H_{1}\right)} s^{c\left(H_{2}\right)} n^{c\left(H_{3}\right)}+r^{c\left(H_{1}\right)} s^{c\left(H_{3}\right)} n^{c\left(H_{2}\right)}+r^{c\left(H_{2}\right)} s^{c\left(H_{1}\right)} n^{c\left(H_{3}\right)} \\
+ & r^{c\left(H_{2}\right)} s^{c\left(H_{3}\right)} n^{c\left(H_{1}\right)}+r^{c\left(H_{3}\right)} s^{c\left(H_{1}\right)} n^{c\left(H_{2}\right)}+r^{c\left(H_{3}\right)} s^{c\left(H_{2}\right)} n^{c\left(H_{1}\right)}
\end{aligned}
$$

to the sum in Lemma 1, which is symmetric. We conclude that $f_{m}(r, s, n)$ is the sum of symmetric polynomials, and is also symmetric. 


\subsection{A simplified equation}

For a 4-edge-colored graph $(G, \delta)$, with possible dotted, dashed, dash-dotted and solid edges, let $|G|$ be the number of vertices in $G$, let $|E(G)|$ be the number of edges in $G$, and let $b(\delta)$ be the number of solid edges in $\delta$. There are $4^{|b(\delta)|}$ sets $V \subseteq C_{m}$ for which $(G, \delta)=(G, \delta)_{V}$, since a solid edge can be formed in 4 possible ways: (a) when exactly two of properties I, II and III hold, or (b) when all three of properties I, II and III hold. From Lemmas 1 and 3, we have

$$
\begin{aligned}
& f_{m}(r, s, n)=\sum_{V \subseteq C_{m}}(-1)^{|V|}\left|\mathcal{B}_{V}\right| \\
& =\sum_{\substack{(G, \delta) \\
|G|=m}} \sum_{\begin{array}{c}
V \subseteq C_{m}: \\
G(\bar{V})=G, \\
\delta(V)=\delta
\end{array}}(-1)^{|V|}\left|\mathcal{B}_{V}\right| \\
& =\sum_{\substack{(G, \delta) \\
|G|=m}} r^{c\left(H_{1}\right)} s^{c\left(H_{2}\right)} n^{c\left(H_{3}\right)} \sum_{\substack{V \subseteq C_{m}: \\
G(\bar{V})=G, \delta(V)=\delta}}(-1)^{|V|} .
\end{aligned}
$$

From here, we use the following identity from [66]: For any $(G, \delta)$, we have

$$
\begin{aligned}
\sum_{\substack{V \subset C_{m}: \\
G(\bar{V})=G, \delta(V)=\delta}}(-1)^{|V|} & =\sum_{x \geqslant 0}\left(\begin{array}{c}
b(\delta) \\
x
\end{array}\right)(-1)^{|E(G)|+b(\delta)+x} 3^{b(\delta)-x} \\
& =(-1)^{|E(G)|}(-2)^{b(\delta)}
\end{aligned}
$$

using the Binomial Theorem. The local variable $x$ counts the number of solid edges where I, II and III all hold. This yields the following theorem:

Theorem 5. For all $r, s, n, m \geqslant 1$, we have

$$
f_{m}(r, s, n)=\sum_{\substack{(G, \delta) \\|G|=m}}(-1)^{|E(G)|}(-2)^{b(\delta)} r^{c\left(H_{1}\right)} s^{c\left(H_{2}\right)} n^{c\left(H_{3}\right)} .
$$

The advantage of Theorem 5 is that it eliminates the need for accounting for clashes (via the variable $V$ ). Instead, we are now working solely with graphs. For computational purposes, it is easier to work with isomorphism classes of graphs (rather than labeled graphs).

We will also account for isolated vertices. For $v \geqslant 0$ and $e \geqslant 0$, let $\Gamma_{e, v}$ denote the set of unlabeled $e$-edge $v$-vertex graphs without isolated vertices (the set $\Gamma_{0,0}$ contains the empty graph, whereas $\left.\Gamma_{e, 1}=\varnothing\right)$. We can split Theorem 5 according to $e, v$, and $\Gamma_{e, v}$ to give the following theorem. 
Theorem 6. For all $r, s, n, m \geqslant 1$, we have

$$
f_{m}(r, s, n)=(r s n)^{m}+\sum_{v \geqslant 2}\left(\begin{array}{c}
m \\
v
\end{array}\right)(r s n)^{m-v+1} \sum_{e \geqslant 1}(-1)^{e} \sum_{G \in \Gamma_{e, v}} \frac{v !}{|\operatorname{Aut}(G)|} P(G)
$$

where

$$
P(G)=P(G ; r, s, n):=\sum_{\delta}(-2)^{b(\delta)} r^{c\left(H_{1}\right)-1} s^{c\left(H_{2}\right)-1} n^{c\left(H_{3}\right)-1}
$$

where the sum is over all edge colorings $\delta$ of $G$.

Proof. Given a graph $G \in \Gamma_{e, v}$, there are $\left(\begin{array}{c}m \\ v\end{array}\right) \frac{v !}{|\operatorname{Aut}(G)|}$ labeled graphs on the vertex set $[m]$ that are isomorphic to $G$ together with $m-v$ isolated vertices. Thus,

$$
\begin{aligned}
f_{m}(r, s, n) & =\sum_{\substack{(G, \delta) \\
|G|=m}}(-1)^{|E(G)|}(-2)^{b(\delta)} r^{c\left(H_{1}\right)} s^{c\left(H_{2}\right)} n^{c\left(H_{3}\right)} \\
& =\sum_{\substack{G \in \Gamma_{e}, v \\
v \geqslant 0 \\
e \geqslant 0}} \sum_{\delta}\left(\begin{array}{c}
m \\
v
\end{array}\right) \frac{v !}{|\operatorname{Aut}(G)|}(-1)^{e}(-2)^{b(\delta)}(r s n)^{m-v} r^{c\left(H_{1}\right)} s^{c\left(H_{2}\right)} n^{c\left(H_{3}\right)} .
\end{aligned}
$$

We obtain the theorem by rearranging this equation.

The following corollary follows straightforwardly from Theorem 6 .

Corollary 7. For fixed $m \geqslant 1$, the polynomial $f_{m}(r, s, n)$ is divisible by $r$ sn.

Furthermore, we use the next result to reduce the required computation.

Lemma 8. Let $G_{1}$ and $G_{2}$ be two graphs. Then,

1. if the two graphs are disjoint, then, $P\left(G_{1} \cup G_{2}\right)=\operatorname{rsn} P\left(G_{1}\right) P\left(G_{2}\right)$; and

2. if the two graphs meet at a single vertex, then $P\left(G_{1} \cup G_{2}\right)=P\left(G_{1}\right) P\left(G_{2}\right)$.

Finally, the following lemma is useful for finding which graphs have to be included when computing the leading terms in $f_{m}(r, s, n)$.

Lemma 9. For any graph $G$ on $v$ vertices, the degree of $(r s n)^{m-v+1} P(G)$ in Theorem 6 is at most $3 m-2 v+2 c(G)$.

Proof. From Theorem 6, the degree of $(r s n)^{m-v+1} P(G)$ is at most $3 m-3 v+\max _{\delta}\left(c\left(H_{1}\right)+\right.$ $\left.c\left(H_{2}\right)+c\left(H_{3}\right)\right)$. Let us show, by induction on the number of edges, that

$$
c\left(H_{1}\right)+c\left(H_{2}\right)+c\left(H_{3}\right) \leqslant v+2 c(G)
$$

for any 4-edge-coloring $\delta$ (with equality when all the edges are dotted, say). If $G$ has no edges, then we have equality in (1). Next, assume (1) holds for some 4-edge-coloring, and 
add a colored edge $x y$. Adding this edge will not increase $c\left(H_{1}\right), c\left(H_{2}\right)$, and $c\left(H_{3}\right)$, so (1) continues to hold unless possibly if adding $x y$ affects $c(G)$. Adding $x y$ decreases $c(G)$ by 1 if and only if $x$ and $y$ belong to separate components of $G$. In this case, $x$ and $y$ also belong to separate components of $H_{1}, H_{2}$, and $H_{3}$. If $x y$ is a dotted edge, then $c\left(H_{2}\right)$ and $c\left(H_{3}\right)$ both decrease by 1 , and (1) holds for the new graph. The same argument works if $x y$ is a dashed or dash-dotted edge. If $x y$ is a solid edge, then $c\left(H_{1}\right), c\left(H_{2}\right)$, and $c\left(H_{3}\right)$ all decrease by 1 , and (1) holds for the new graph.

\section{Chromatic polynomial method}

Let $R_{r, s}$ be the $r \times s$ rook's graph, i.e., the Cartesian product of $K_{r}$ and $K_{s}$. The graph $R_{3,4}$ is drawn in Figure 2. Any partial Latin rectangle in $\operatorname{PLR}(r, s, n ; m)$ can be interpreted as a proper $n$-coloring of an $m$-vertex induced subgraph of $R_{r, s}$. An example of this correspondence is also given in Figure 2.
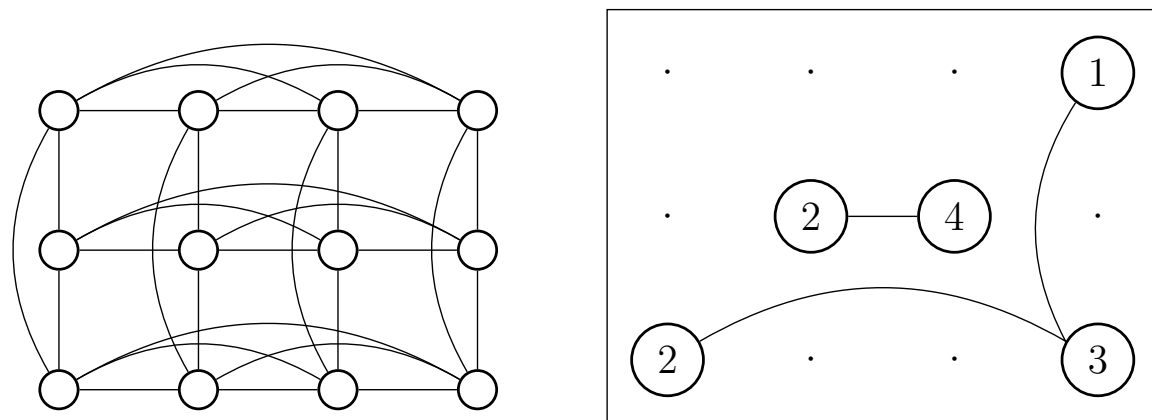

Figure 2: The graph $R_{3,4}$ along with a proper 4-coloring of an induced 5-vertex subgraph of $R_{3,4}$. This illustrates the corresponding partial Latin rectangle in $\operatorname{PLR}(3,4,4 ; 5)$.

We can naturally think of (labeled) induced subgraphs of $R_{r, s}$ as $(0,1)$-matrices, with a 1 in cell $(i, j)$ whenever vertex $(i, j)$ is present. Under this equivalence, we talk of the rows and columns of such graphs, and of the chromatic polynomial of their corresponding induced subgraphs. If $\Pi$ denotes this chromatic polynomial, then

$$
\# \operatorname{PLR}(r, s, n ; m)=\sum_{M} \Pi(M ; n)
$$

where the sum is over all $r \times s(0,1)$-matrices $M$ with exactly $m$ ones (or equivalently over all $m$-vertex induced subgraphs of $\left.R_{r, s}\right)$.

Given any $r \times s(0,1)$-matrix $M$, we define a general block of $M$ to be a submatrix $H$ in which: (a) every row and every column of $H$ contains a 1 ; (b) in $M$, there are no 1 's in the rows of $H$ outside of $H$; and (c) in $M$, there are no 1's in the columns of $H$ outside of $H$. We define a block as a general block which has no proper submatrix which is a general block in itself. Blocks correspond to components of the induced subgraph of $R_{r, s}$. 
We act on the set of $r \times s(0,1)$-matrices by permuting rows and columns. Under this action, we choose representatives from each orbit and call them canonical. After that, we define a function $C$ such that $C(M)$ is the canonical matrix in the orbit of each $(0,1)$ matrix $M$. From $M$, we can also construct a multiset $\left\{C\left(M_{i}\right)\right\}_{i=1}^{k}$ where $M_{1}, M_{2}, \ldots, M_{k}$ are the blocks of $M$.

In the other direction, let $\mathcal{K}_{r, s, m, k}$ denote the set of multisets $\mathbf{K}=\left\{K_{i}\right\}_{i=1}^{k}$ of canonical blocks such that (a) the number of 1's in the blocks sum to $m$; (b) the number of rows in the blocks sum to $\leqslant r$; and (c) the number of columns in the blocks sum to $\leqslant s$. Given any $\mathbf{K} \in \mathcal{K}_{r, s, m, k}$, we can arrange the blocks as follows:

\begin{tabular}{|c|c|c|c|c|}
\hline$K_{1}$ & $\varnothing$ & $\cdots$ & $\varnothing$ & $\varnothing$ \\
\hline$\varnothing$ & $K_{2}$ & $\cdots$ & $\varnothing$ & $\varnothing$ \\
\hline$\vdots$ & $\vdots$ & $\ddots$ & $\vdots$ & $\vdots$ \\
\hline$\varnothing$ & $\varnothing$ & $\cdots$ & $K_{k}$ & $\varnothing$ \\
\hline$\varnothing$ & $\varnothing$ & $\cdots$ & $\varnothing$ & $\varnothing$ \\
\hline
\end{tabular}

where $\varnothing$ denotes an all-0 submatrix, so that there are $r$ rows and $c$ columns. Call this matrix $M(\mathbf{K})$. If we permute the rows and columns of this matrix, we generate every $r \times s(0,1)$-matrix $M$ that has $\left\{C\left(M_{i}\right)\right\}_{i=1}^{k}=\mathbf{K}$ some number of times, $\Gamma_{\mathbf{K}}$ say, by the Orbit-Stabilizer Theorem. It follows from (2) that

$$
\# \operatorname{PLR}(r, s, n ; m)=\sum_{k \geqslant 0} \sum_{\mathbf{K} \in \mathcal{K}_{r, s, m, k}} \frac{r ! s !}{\Gamma_{\mathbf{K}}} \Pi(M(\mathbf{K}) ; n) .
$$

If $\mathbf{K}=\left\{K_{i}\right\}_{i=1}^{k}$, then

$$
\Pi(M(\mathbf{K}) ; n)=\prod_{i=1}^{k} \Pi\left(K_{i} ; n\right)
$$

since each $K_{i}$ corresponds to a disjoint component in the induced subgraph of $R_{r, s}$.

Let $e_{\text {row }}$ and $e_{\text {col }}$ denote the number of non-empty rows and columns in the matrix $M(\mathbf{K})$, respectively. If there are $\ell$ distinct matrices in the multiset $\mathbf{K}$, let $k_{i}$, for $i \in[\ell]$, be the number of copies of the $i$-th distinct matrix. The elements in the stabilizer of $M(\mathbf{K})$ are those which permute the all-0 rows and columns, permute the identical blocks amongst themselves, and stabilize each $K_{i}$ individually. Thus since every $K_{i} \in \mathbf{K}$ is canonical,

$$
\Gamma_{\mathbf{K}}=\left(r-e_{\text {row }}\right) !\left(s-e_{\text {col }}\right) !\left(\prod_{i=1}^{k}\left|\operatorname{Stab}\left(K_{i}\right)\right|\right)\left(\prod_{i=1}^{\ell} k_{i} !\right) .
$$

The stabilizer of a $(0,1)$-matrix $M=\left(M_{i j}\right)$ under row and column permutations is isomorphic to the automorphism group of the vertex-colored bipartite graph $G_{M}$ with vertex set

$$
\overbrace{\left\{\mathfrak{r}_{1}, \ldots, \mathfrak{r}_{r}\right\}}^{\text {color } 1} \cup \overbrace{\left\{\mathfrak{c}_{1}, \ldots, \mathfrak{c}_{s}\right\}}^{\text {color } 2}
$$

and edges $\mathfrak{r}_{i} \mathfrak{c}_{j}$ if and only if $M_{i j}=1$.

To compute \#PLR $(r, s, n ; m)$ for small $m$, we thus: 
- Generate a list of possible blocks $K$ with up to $m$ ones, inequivalent under row and column permutations, and compute, for each block, the size of $\operatorname{Aut}\left(G_{K}\right)$, and the chromatic polynomial of $K$. Table 1 lists the results of this computation for $m \leqslant 5$.

- Iterate through each $\mathbf{K} \in \cup_{k \geqslant 0} \mathcal{K}_{r, s, m, k}$, computing its contribution to (3) from the table generated in the first step.

To further reduce the computation, we only store blocks with no more rows than columns. This requires the modification of (4) to account for transposing the blocks. By ordering the set of all blocks, a multiset $\mathbf{K}=\left\{K_{i}\right\}_{i=1}^{k}$ is equivalent to a unique ordered sequence $\left(K_{i}\right)_{i=1}^{k}$. We use a $(0,1)$-sequence $\left(t_{i}\right)_{i=1}^{k}$ to keep track of which $K_{i}$ we transpose, with 1 meaning "transpose" and 0 meaning "don't transpose". We define $\left(t_{i}\right)_{i=1}^{k}$ as good if (a) $t_{i}=0$ whenever $K_{i}$ is a square matrix, and (b) if $K_{i}=K_{i+1}$ and $t_{i}=0$, then $t_{i+1}=0$. We choose not to transpose square matrices at this stage, as it adds the task of identifying when the transpose of a matrix can be formed by permuting its rows and columns, hence we have condition (a). Condition (b) prevents overcounting in cases such as

\begin{tabular}{|lll}
1 & 0 & 0 \\
1 & 0 & 0 \\
0 & 1 & 1
\end{tabular}$\quad$ and $\quad$\begin{tabular}{lll|}
1 & 1 & 0 \\
0 & 0 & 1 \\
0 & 0 & 1 \\
\hline
\end{tabular}

Define

$$
\overline{K_{i}}= \begin{cases}K_{i} & \text { if } t_{i}=0 \\ \left(K_{i}\right)^{T} & \text { if } t_{i}=1\end{cases}
$$

Thus, (3) can be rephrased to give the following theorem.

Theorem 10. For all $m \geqslant 1$, we have

$$
\# \operatorname{PLR}(r, s, n ; m)=\sum_{k \geqslant 0} \sum_{\mathbf{K} \in \mathcal{K}_{r, s, m, k}} \sum_{\substack{\left(t_{i}\right)_{i=1}^{k} \\ \text { good }}}[r]_{e_{\text {row }}}[s]_{e_{\mathrm{col}}} \frac{\prod_{i=1}^{k} \Pi\left(\overline{K_{i}} ; n\right)}{\left(\prod_{i=1}^{k}\left|\operatorname{Aut}\left(G_{K_{i}}\right)\right|\right)\left(\prod_{i=1}^{\ell} k_{i} !\right)}
$$

where $[r]_{e_{\text {row }}}=r ! /\left(r-e_{\text {row }}\right) !$ and $[s]_{e_{\mathrm{col}}}=s ! /\left(s-e_{\mathrm{col}}\right) !$.

\section{Sade's method}

Sade's method [58] outstrips all other methods for finding the number of Latin squares [61]. Subsequent authors $[10,53,52,74]$ who found the number of Latin squares of orders $n \in\{8,9,10,11\}$ implemented optimized computerized versions of Sade's method. We generalize Sade's method to partial Latin rectangles: 
Lemma 11. Let $L, M \in \operatorname{PLR}(r, s, n)$. If

1. L and $M$ have the same set of symbols in each column, or

2. $L$ is isotopic to $M$,

then they can be extended in the same number of ways to $(r+1) \times s$ partial Latin rectangles of a given weight $m$ by adding an $(r+1)$-th row.

Proof. In the first case, the possible $(r+1)$-th rows for $L$ and $M$ are the same. In the second case, if $M=L^{\Theta}$, then the possible $(r+1)$-th rows of $L^{\Theta}$ are precisely the possible $(r+1)$-th rows of $L$ after applying $\Theta$.

Let $L, M \in \operatorname{PLR}(r, s, n)$. We say that $L$ and $M$ are Sade equivalent if $L$ is isotopic to a partial Latin rectangle $L^{\prime}$ such that the columns of $L^{\prime}$ and $M$ have the same sets of symbols. Particularly, $L$ and $M$ must have the same weight. Thus, for instance, the following four partial Latin rectangles in $\operatorname{PLR}(2,3,3 ; 4)$ are Sade equivalent.

\begin{tabular}{|lll}
1 & 3 & 2 \\
$\cdot$ & 2 & $\cdot$
\end{tabular},$\quad \begin{array}{lll}3 & 1 & 2 \\
2 & \cdot & \cdot\end{array}, \quad, \quad \begin{array}{ccc}1 & 3 & 2 \\
2 & \cdot & \cdot\end{array} \quad$ and $\quad \begin{array}{lll}2 & 3 & \cdot \\
1 & \cdot & 2\end{array}$.

Practically, we need a fast method for checking whether a large number of partial Latin rectangles are Sade equivalent. To this end, for each partial Latin rectangle $L \in \operatorname{PLR}(r, s, n)$, we perform the following steps, which we illustrate for this example $\operatorname{PLR}(3,4,3 ; 6)$ :

$$
\begin{array}{|cccc|}
\hline 1 & \cdot & 2 & \cdot \\
2 & 1 & 3 & \cdot \\
\cdot & \cdot & \cdot & 3 \\
\hline
\end{array}
$$

1. Construct a vertex-colored bipartite graph with vertex set

$$
\overbrace{\left\{\mathfrak{c}_{1}, \ldots, \mathfrak{c}_{s}\right\}}^{\text {color } 1} \cup \overbrace{\left\{\mathfrak{n}_{1}, \ldots, \mathfrak{n}_{n}\right\}}^{\text {color } 2}
$$

and edge set

$$
\left\{\mathfrak{c}_{i} \mathfrak{n}_{j} \text { : symbol } j \text { occurs in column } i \text { in } L\right\} .
$$

In our running example (5), we obtain

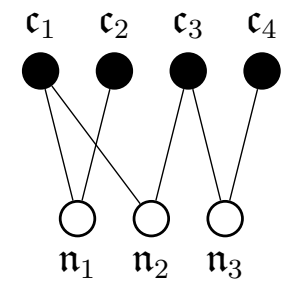


2. Canonically label the graph in a way that preserves the vertex colors (to this end we use nauty [49], for which such a labeling is an internal procedure). In our running example, we obtain

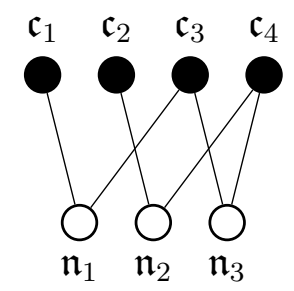

3. Find the submatrix of the adjacency matrix formed by the rows indexed by $\left\{\mathfrak{c}_{i}\right\}_{i=1}^{s}$ and columns indexed $\left\{\mathfrak{n}_{i}\right\}_{i=1}^{n}$, and read it as a binary number. We call the result the Sade number, denoted $s n_{L}$, of the partial Latin rectangle $L$. In our running example, we obtain

\begin{tabular}{|lllllll|}
\hline 0 & 0 & 0 & 0 & 1 & 0 & 0 \\
0 & 0 & 0 & 0 & 0 & 1 & 0 \\
0 & 0 & 0 & 0 & 1 & 0 & 1 \\
0 & 0 & 0 & 0 & 0 & 1 & 1 \\
1 & 0 & 1 & 0 & 0 & 0 & 0 \\
0 & 1 & 0 & 1 & 0 & 0 & 0 \\
0 & 0 & 1 & 1 & 0 & 0 & 0 \\
\hline
\end{tabular}

which gives the Sade number of (5) as 100010101011 in binary, or 2219 in decimal.

We describe how to implement Sade's method for partial Latin rectangles in Algorithm 1. Along with a partial Latin rectangle $L$ itself, we store its Sade number $s n_{L}$ and the number of Sade equivalent partial Latin rectangles, which we call the Sade multiplier $s m_{L}$. For each $i \in\{0,1, \ldots, s\}$, we maintain a database of Sade inequivalent $i \times s$ partial Latin rectangles $P L R s[i]$. We compute $P L R s[i]$ by extending the partial Latin rectangles in $P L R s[i-1]$ in all possible ways, then filtering out Sade equivalent partial Latin rectangles.

Importantly, PLRs $[i]$ is sorted according to Sade numbers. This enables the use of binary search when checking for equivalent partial Latin rectangles, thereby greatly reducing the number of pairwise equivalence comparisons we need to make. We iterate through extensions of partial Latin rectangles by use of a backtracking algorithm.

Other practical improvements can be made:

- In enumerating up to $8 \times 8$ partial Latin rectangles, the Sade number will be less than $2^{64}$, which can thus be stored as 64-bit unsigned integers.

- When processing the last few rows, we can forgo Sade's method and instead use a simple backtracking algorithm to count the number of extensions up to completions of each partial Latin rectangle.

- Although \#PLR $(r, s, n ; m)=\# \operatorname{PLR}(r, n, s ; m)$, it is significantly faster to compute the value of $\# \operatorname{PLR}(r, s, n ; m)$ when $s \leqslant n$. 


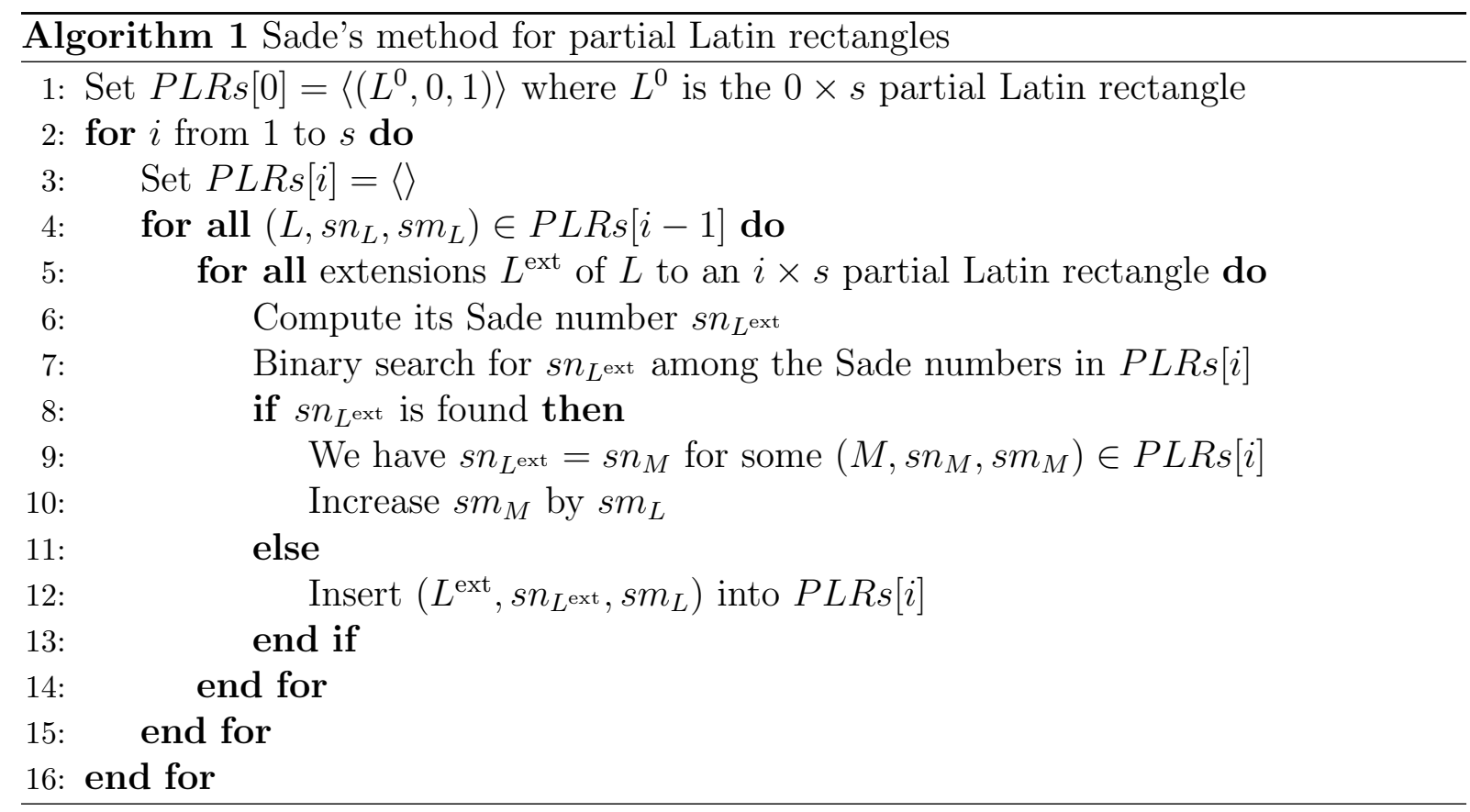

\section{$5 \quad$ Algebraic geometry method}

In this section, we review how sets of partial Latin rectangles are identified with the algebraic sets of certain ideals. This follows the idea of Bayer [11] and Adams and Loustaunau [2] to solve the problem of $n$-coloring a graph by means of algebraic geometry, since every Latin square of order $n$ is equivalent to an $n$-colored bipartite graph $K_{n, n}$ [47]. Much more recently, this algebraic method has been adapted to solve sudokus [7, 37, 59], enumerate quasigroup rings derived from partial Latin squares [22], enumerate partial Latin rectangles that admit a given autotopism [27, 28, 30, 32] or autoparatopism [36], and also the number of isotopisms between two given partial Latin rectangles [34], thereby enabling us to compute in Section 6 the numbers of equivalence classes by means of the OrbitStabilizer Theorem and Burnside's Lemma. See $[16,46]$ for more details on algebraic geometry.

Let $\mathbb{K}[\mathbf{x}]=\mathbb{K}\left[x_{1}, \ldots, x_{n}\right]$ be a polynomial ring in $n$ variables over a field $\mathbb{K}$. An ideal of $\mathbb{K}[\mathbf{x}]$ is any subset $I \subseteq \mathbb{K}[\mathbf{x}]$ that (a) contains the zero polynomial; (b) is closed under polynomial addition; and (c) is closed under multiplication by polynomials in $q \in \mathbb{K}[\mathbf{x}]$. The ideal generated by a finite set of polynomials $\left\{p_{1}, \ldots, p_{m}\right\} \subseteq \mathbb{K}[\mathbf{x}]$ is defined as

$$
\left\langle p_{1}, \ldots, p_{m}\right\rangle:=\left\{p \in \mathbb{K}[\mathbf{x}]: p=\sum_{i=1}^{m} q_{i} p_{i}, \text { where } q_{i} \in \mathbb{K}[\mathbf{x}] \text {, for all } i \leqslant m\right\} \text {. }
$$

The algebraic set of $I$ is the set of points

$$
\mathcal{V}(I):=\left\{\left(a_{1}, \ldots, a_{n}\right) \in \mathbb{K}^{n}: p\left(a_{1}, \ldots, a_{n}\right)=0, \text { for all } p \in I\right\} .
$$

For this paper, interest in this topic arises from the polynomial ring

$$
\mathbb{Q}[\mathbf{x}]=\mathbb{Q}\left[x_{111}, \ldots, x_{r s n}\right]
$$


and the ideal

$$
\begin{aligned}
I_{r, s, n ; m}:= & \left\langle x_{i j k}^{2}-x_{i j k}:(i, j, k) \in[r] \times[s] \times[n]\right\rangle \\
& +\left\langle x_{i j k} x_{i^{\prime} j k}:(i, j, k) \in[r] \times[s] \times[n], i^{\prime} \in[r], i<i^{\prime}\right\rangle \\
& +\left\langle x_{i j k} x_{i j^{\prime} k}:(i, j, k) \in[r] \times[s] \times[n], j^{\prime} \in[s], j<j^{\prime}\right\rangle \\
& +\left\langle x_{i j k} x_{i j k^{\prime}}:(i, j, k) \in[r] \times[s] \times[n], k^{\prime} \in[n], k<k^{\prime}\right\rangle \\
& +\left\langle m-\sum_{i \in[r]} \sum_{j \in[s]} \sum_{k \in[n]} x_{i j k}\right\rangle .
\end{aligned}
$$

There is a bijection between partial Latin rectangles $L=\left(l_{i j}\right) \in \operatorname{PLR}(r, s, n ; m)$ and elements of the algebraic set of $I_{r, s, n ; m}$ : we have $l_{i j}=k$ whenever $x_{i j k}=1$, and $l_{i j}$ is undefined otherwise. More specifically:

- Having $x_{i j k}^{2}-x_{i j k}=0$ implies that the algebraic set is contained in $\{0,1\}^{r s n}$.

- Having $x_{i j k} x_{i^{\prime} j k}=0$ implies that the symbol $k$ does not appear twice in the column $j$.

- Having $x_{i j k} x_{i j^{\prime} k}=0$ implies that the symbol $k$ does not to appear twice in the row $i$.

- Having $x_{i j k} x_{i j k^{\prime}}=0$ implies that there is at most one symbol in the cell $(i, j)$.

- Having $\sum_{i \in[r]} \sum_{j \in[s]} \sum_{k \in[n]} x_{i j k}=m$ implies that the weight of the partial Latin rectangle $L$ is $m$.

Since the algebraic set $\mathcal{V}\left(I_{r, s, n ; m}\right)$ is finite and the ideal $I_{r, s, n ; m} \cap \mathbb{Q}\left[x_{i j k}\right]$ is generated by the polynomial $x_{i j k}^{2}-x_{i j k}$, which is contained in $I_{r, s, n ; m}$, Seidenberg's Lemma and [46, Theorem 3.7.19] imply

$$
\# \operatorname{PLR}(r, s, n ; m)=\left|\mathcal{V}\left(I_{r, s, n ; m}\right)\right|=\operatorname{dim}_{\mathbb{Q}}\left(\mathbb{Q}[\mathbf{x}] / I_{r, s, n ; m}\right) .
$$

This fact has recently been used in [30] to compute \#PLR $(r, s, n ; m)$, for all $r, s, n \leqslant 6$ and $m \leqslant r s$.

This algebraic geometry enumeration method can be generalized to include cases in which a certain autoparatopism is imposed as follows.

Theorem 12. Let $\Theta=\left(\delta_{1}, \delta_{2}, \delta_{3}\right) \in \mathfrak{I}_{r, s, n}$ and $\pi \in S_{3}$. Define

$$
I_{(\Theta, \pi) ; m}:=I_{r, s, n ; m}+\left\langle x_{i_{1} i_{2} i_{3}}-x_{\delta_{\pi(1)}\left(i_{\pi(1)}\right) \delta_{\pi(2)}\left(i_{\pi(2)}\right) \delta_{\pi(3)}\left(i_{\pi(3)}\right)}: i_{1} \in[r], i_{2} \in[s], i_{3} \in[n]\right\rangle .
$$

Then, the set $\operatorname{PLR}((\Theta, \pi) ; m)$ has a natural bijection with $\mathcal{V}\left(I_{(\Theta, \pi) ; m}\right)$ and

$$
\# \operatorname{PLR}((\Theta, \pi) ; m)=\operatorname{dim}_{\mathbb{Q}}\left(\mathbb{Q}[\mathbf{x}] / I_{(\Theta, \pi) ; m}\right) .
$$


Proof. Since $\mathcal{V}\left(I_{(\Theta, \pi) ; m}\right) \subseteq \mathcal{V}\left(I_{r, s, n ; m}\right)$, any point in $\mathcal{V}\left(I_{(\Theta, \pi) ; m}\right)$ corresponds to a partial Latin rectangle $L=\left(l_{i_{1} i_{2}}\right) \in \operatorname{PLR}(r, s, n ; m)$ as previously described. The addition of the polynomials $x_{i_{1} i_{2} i_{3}}-x_{\delta_{\pi(1)}\left(i_{\pi(1)}\right) \delta_{\pi(2)}\left(i_{\pi(2)}\right) \delta_{\pi(3)}\left(i_{\pi(3)}\right)}$ implies that if $l_{i_{1} i_{2}}$ is defined and $l_{i_{1} i_{2}}=i_{3}$, then $l_{\delta_{\pi(1)}\left(i_{\pi(1)}\right) \delta_{\pi(2)}\left(i_{\pi(2)}\right)}=\delta_{\pi(3)}\left(i_{\pi(3)}\right)$. Thus, $(\Theta, \pi)$ is an autoparatopism of $L$.

A similar approach enables us to determine the set of isotopisms between two partial Latin rectangles as follows.

Theorem 13. Let $\mathbb{Q}[\mathbf{x}]=\mathbb{Q}\left[x_{11}, \ldots, x_{r r}, y_{11}, \ldots, y_{s s}, z_{11}, \ldots, z_{n n}\right]$ be a polynomial ring in $r^{2}+s^{2}+n^{2}$ variables. The set $\mathfrak{I}(P, Q)$ of isotopisms between two partial Latin rectangles $P=\left(p_{i j}\right)$ and $Q=\left(q_{i j}\right)$ in $\operatorname{PLR}(r, s, n)$ has a natural bijection with the algebraic set of the ideal

$$
\begin{aligned}
I_{P, Q}= & \left\langle x_{i j}^{2}-x_{i j}: i, j \in[r]\right\rangle+\left\langle y_{i j}^{2}-y_{i j}: i, j \in[s]\right\rangle+\left\langle z_{i j}^{2}-z_{i j}: i, j \in[n]\right\rangle \\
& +\left\langle 1-\sum_{j \in[r]} x_{i j}: i \in[r]\right\rangle+\left\langle 1-\sum_{i \in[r]} x_{i j}: j \in[r]\right\rangle \\
& +\left\langle 1-\sum_{j \in[s]} y_{i j}: i \in[s]\right\rangle+\left\langle 1-\sum_{i \in[s]} y_{i j}: j \in[s]\right\rangle \\
& +\left\langle 1-\sum_{j \in[n]} z_{i j}: i \in[n]\right\rangle+\left\langle 1-\sum_{i \in[n]} z_{i j}: j \in[n]\right\rangle \\
& +\left\langle x_{i k} y_{j l}\left(z_{p_{i j} q_{k l}}-1\right): i, k \in[r], j, l \in[s], \text { such that } p_{i j}, q_{k l} \in[n]\right\rangle \\
& +\left\langle x_{i k} y_{j l}: i, k \in[r], j, l \in[s], p_{i j} \text { and/or } q_{k l} \text { is undefined }\right\rangle .
\end{aligned}
$$

Consequently, the number of isotopisms from $P$ to $Q$ is given by

$$
\# \mathfrak{I}(P, Q)=\operatorname{dim}_{\mathbb{Q}}\left(\mathbb{Q}[\mathbf{x}] / I_{P, Q}\right) .
$$

Proof. The first three subideals of $I_{P, Q}$ imply $\mathcal{V}\left(I_{P, Q}\right) \subseteq\{0,1\}^{r^{2}+s^{2}+n^{2}}$. Every isotopism $\Theta=(\alpha, \beta, \gamma) \in \mathfrak{I}_{r, s, n}$ uniquely corresponds to a zero $\left(x_{11}^{\Theta}, \ldots, x_{r r}^{\Theta}, y_{11}^{\Theta}, \ldots, y_{s s}^{\Theta}, z_{11}^{\Theta}, \ldots, z_{n n}^{\Theta}\right)$ in $\mathcal{V}\left(I_{P, Q}\right)$, where $x_{i j}^{\Theta}=1$, (resp., $y_{i j}^{\Theta}=1$ and $z_{i j}^{\Theta}=1$ ) if $\alpha(i)=j$ (resp., $\beta(i)=j$ and $\gamma(i)=j$ ) and 0 , otherwise. Specifically, the fourth and fifth subideals of $I_{P, Q}$ (in the statement of Theorem 13) imply $\alpha$ is a permutation of $S_{r}$ (the fourth one ensures the injectivity, while the fifth one ensures surjectivity). Similarly, the next two pairs of subideals imply $\beta$ and $\gamma$ are permutations of $S_{s}$ and $S_{n}$, respectively, and hence, $\Theta$ is an isotopism of $\operatorname{PLR}(r, s, n)$. The last two subideals imply $\Theta$ is a bijection between the entry sets $E(P)$ and $E(Q)$. Further, since $I_{P, Q} \cap \mathbb{Q}\left[x_{i j k}\right]=\left\langle x_{i j k}^{2}-x_{i j k}\right\rangle \subseteq I_{P, Q}$, Seidenberg's Lemma and Theorem 3.7.19 in [46] imply the theorem statement.

\section{Counting equivalence classes of partial Latin rectangles}

Theorem 13 can be used to determine not only the size of the autotopism group $\mathfrak{I}(P, P)$ of a partial Latin rectangle $P$, but also that of its autoparatopism group $\mathfrak{P}(P, P)$, because

$$
\# \mathfrak{P}(P, P)=\sum_{\pi \in S_{3}} \# \mathfrak{I}\left(P, P^{\pi}\right) .
$$


The following result shows how the computation of both values enables us to determine the size of the isotopism and main classes containing $P$ by means of the Orbit-Stabilizer Theorem.

Theorem 14. Let $P \in \operatorname{PLR}(r, s, n)$. Then,

1. the number of partial Latin rectangles that are isotopic to $P$, i.e., the size of the isotopism class containing $P$, is

$$
\frac{r ! s ! n !}{\# \mathfrak{I}(P, P)}
$$

2. the number of partial Latin rectangles that are paratopic to $P$, i.e., the size of the main class containing $P$, is

$$
\# S_{r, s, n} \frac{r ! s ! n !}{\# \mathfrak{P}(P, P)}
$$

and

3. the number of isotopism classes in the main class of $P$ is

$$
\# S_{r, s, n} \frac{\# \mathfrak{I}(P, P)}{\# \mathfrak{P}(P, P)} .
$$

Proof. The first two claims follow from the Orbit-Stabilizer Theorem. For the third claim, we observe that paratopic partial Latin rectangles have autotopism groups of the same size, because $\Theta$ is an autotopism of $P$ if and only if $\Lambda^{-1} \Theta \Lambda$ is an autotopism of $P^{\Lambda}$ for any paratopism $\Lambda$. They thus also have isotopism classes of the same size, which partition the main class, so the first two claims imply the third.

From here on, let $\operatorname{Isom}(n ; m)$, Isot $(r, s, n ; m)$ and $\operatorname{MC}(r, s, n ; m)$ respectively denote the set of isomorphism classes of $\operatorname{PLS}(n ; m)$ and the sets of isotopism and main classes of $\operatorname{PLR}(r, s, n ; m)$. The following result follows straightforwardly from Burnside's Lemma and letting the isomorphism, isotopism, and paratopism groups act on the set of partial Latin rectangles of a given order.

Theorem 15. Let $r, s, n \geqslant 1$ and $m \leqslant r s$. Then,

1. the number of isomorphism classes in $\operatorname{PLS}(n ; m)$ is

$$
\# \operatorname{Isom}(n ; m)=\frac{1}{n !} \sum_{\pi \in S_{n}} \# \operatorname{PLR}(((\pi, \pi, \pi), \mathrm{Id}) ; m) \text {; }
$$

2. the number of isotopism classes in $\operatorname{PLR}(r, s, n ; m)$ is

$$
\text { \#Isot }(r, s, n ; m)=\frac{1}{r ! s ! n !} \sum_{\Theta \in \mathfrak{I}_{r, s, n}} \# \operatorname{PLR}((\Theta, \mathrm{Id}) ; m) ;
$$

and 
3. the number of main classes in $\operatorname{PLR}(r, s, n ; m)$ is

$$
\# \mathrm{MC}(r, s, n ; m)=\frac{1}{r ! s ! n ! \# S_{r, s, n}} \sum_{(\Theta, \pi) \in \mathfrak{P}_{r, s, n}} \# \operatorname{PLR}((\Theta, \pi) ; m) .
$$

In practice, it is not necessary to compute all the values $\# \operatorname{PLR}((\Theta, \pi) ; m)$ in order to determine $\# \operatorname{Isom}(n ; m)$, \# $\operatorname{Isot}(r, s, n ; m)$, or \# $\# \mathrm{MC}(r, s, n ; m)$ in Theorem 15. The following lemma implies that \#PLR $((\Theta, \pi) ; m)$ only depends on the conjugacy class of the corresponding paratopism. Recall that two permutations $\alpha, \beta \in S_{n}$ are conjugate if there exists a third permutation $\gamma \in S_{n}$ such that $\alpha=\gamma^{-1} \beta \gamma$, which naturally generalizes to isotopisms and paratopisms under componentwise conjugacy. In what follows, conjugacy is denoted $\sim$.

Lemma 16. Let $\left(\Theta_{1}, \pi_{1}\right)$ and $\left(\Theta_{2}, \pi_{2}\right)$ be two conjugate paratopisms in $\mathfrak{P}_{r, s, n}$. Then,

$$
\# \operatorname{PLR}\left(\left(\Theta_{1}, \pi_{1}\right) ; m\right)=\# \operatorname{PLR}\left(\left(\Theta_{2}, \pi_{2}\right) ; m\right) \text {. }
$$

Proof. Since $\left(\Theta_{1}, \pi_{1}\right) \sim\left(\Theta_{2}, \pi_{2}\right)$, there exists $\left(\Theta_{3}, \pi_{3}\right) \in \mathfrak{P}_{r, s, n}$ such that $\left(\Theta_{2}, \pi_{2}\right)=$ $\left(\Theta_{3}, \pi_{3}\right)^{-1}\left(\Theta_{1}, \pi_{1}\right)\left(\Theta_{3}, \pi_{3}\right)$. Let $L \in \operatorname{PLR}\left(\left(\Theta_{1}, \pi_{1}\right) ; m\right)$. Then,

$$
\left(L^{\left(\Theta_{3}, \pi_{3}\right)}\right)^{\left(\Theta_{2}, \pi_{2}\right)}=\left(L^{\left(\Theta_{1}, \pi_{1}\right)}\right)^{\left(\Theta_{3}, \pi_{3}\right)}=L^{\left(\Theta_{3}, \pi_{3}\right)}
$$

and so, $L^{\left(\Theta_{3}, \pi_{3}\right)} \in \operatorname{PLR}\left(\left(\Theta_{2}, \pi_{2}\right) ; m\right)$. The result holds because $\operatorname{PLR}\left(\left(\Theta_{2}, \pi_{2}\right) ; m\right)=$ $\left\{L^{\left(\Theta_{3}, \pi_{3}\right)}: L \in \operatorname{PLR}\left(\left(\Theta_{1}, \pi_{1}\right) ; m\right)\right\}$.

The following result is shown using similar reasoning to that used by Mendis and Wanless in the proof of Theorem 2.2 in [55] for paratopisms of Latin squares.

Theorem 17. Two paratopisms $\left(\left(\alpha_{1}, \alpha_{2}, \alpha_{3}\right), \pi_{1}\right)$ and $\left(\left(\beta_{1}, \beta_{2}, \beta_{3}\right), \pi_{2}\right)$ in $\mathfrak{P}_{r, s, n}$ are conjugate if and only if there is a length preserving bijection $\eta$ from the cycles of $\pi_{1}$ to those of $\pi_{2}$ such that, if $\eta$ maps a cycle $\left(a_{1}, \ldots, a_{k}\right)$ to a cycle $\left(b_{1}, \ldots, b_{k}\right)$, both of them in the symmetric group $S_{3}$, then $\alpha_{a_{1}} \cdots \alpha_{a_{k}} \sim \beta_{b_{1}} \cdots \beta_{b_{k}}$.

As a consequence, any paratopism $((\alpha, \beta, \gamma),(12)) \in \mathfrak{P}_{r, r, n}$ is conjugate to the paratopism $((\mathrm{Id}, \alpha \beta, \gamma),(12)) \in \mathfrak{P}_{r, r, n}$, and any paratopism $((\alpha, \beta, \gamma),(123)) \in \mathfrak{P}_{r, r, r}$ is conjugate to both paratopisms $((\mathrm{Id}, \mathrm{Id}, \alpha \beta \gamma),(123)) \in \mathfrak{P}_{r, r, r}$ and $((\mathrm{Id}, \mathrm{Id}, \alpha \beta \gamma),(132)) \in \mathfrak{P}_{r, r, r}$.

Proof. If $r=s=n$, or if $\pi_{1}=\pi_{2}$, the proof of Theorem 2.2 in [55] suffices the prove the theorem. Otherwise, up to equivalence, we have $\pi_{1}=(12)$ and $\pi_{2}=\operatorname{Id}$ and $r=s \neq n$. Clearly, $\eta$ does not exist. The two paratopisms are not conjugate since conjugation in $\mathfrak{P}_{r, s, n}$ preserves the conjugacy class of the parastrophe permutation.

Theorem 18. For any paratopism $((\alpha, \beta, \gamma),(12)) \in \mathfrak{P}_{r, r, n}$, we have

$$
\begin{aligned}
& \# \operatorname{PLR}(((\alpha, \beta, \gamma),(12)) ; m)=\# \operatorname{PLR}(((\beta, \alpha, \gamma),(12)) ; m) \\
&=\# \operatorname{PLR}(((\alpha, \gamma, \beta),(13)) ; m)=\# \operatorname{PLR}(((\beta, \gamma, \alpha),(13)) ; m) \\
&=\# \operatorname{PLR}(((\gamma, \alpha, \beta),(23)) ; m)=\# \operatorname{PLR}(((\gamma, \beta, \alpha),(23)) ; m) .
\end{aligned}
$$


Moreover, any $((\alpha, \beta, \gamma),(12)) \in \mathfrak{P}_{r, r, n}$ is conjugate to a paratopism $\left(\left(\mathrm{Id}, \beta^{\prime}, \gamma^{\prime}\right),(12)\right)$, where $\alpha \beta \sim \beta^{\prime}$ and $\gamma \sim \gamma^{\prime}$. If $\pi$ is a 3-cycle, then any paratopism $((\alpha, \beta, \gamma), \pi) \in \mathfrak{P}_{r, r, r}$ is conjugate to a paratopism $((\mathrm{Id}, \mathrm{Id}, \gamma),(123)) \in \mathfrak{P}_{r, s, n}$, where $\alpha \beta \gamma \sim \gamma^{\prime}$.

Proof. We have that $((\alpha, \beta, \gamma),(12))$ is a paratopism of $L$ if and only if $((\alpha, \gamma, \beta),(13))$ is a paratopism of $L^{(23)}$. Consequently,

$$
\# \operatorname{PLR}(((\alpha, \beta, \gamma),(12)) ; m)=\# \operatorname{PLR}(((\alpha, \gamma, \beta),(13)) ; m)
$$

Lemma 16 implies

$$
\# \operatorname{PLR}(((\alpha, \beta, \gamma),(12)) ; m)=\# \operatorname{PLR}(((\beta, \alpha, \gamma),(12)) ; m)
$$

And we similarly prove the other equalities. Theorem 17 implies the conjugacy claims.

Conjugacy in symmetric groups constitutes an equivalence relation in which each conjugacy class is characterized by the common cycle structure of their elements. Recall that the cycle structure of a permutation $\pi \in S_{m}$ is the expression

$$
z_{\pi}:=m^{d_{m}^{\pi}} \cdots 1^{d_{1}^{\pi}}
$$

where $d_{i}^{\pi}$ denotes the number of cycles of length $i$ in the unique cycle decomposition of the permutation $\pi$. Thus, for instance, the cycle structure of the permutation (12)(345)(78)(9) is $3^{1} 2^{2} 1^{1}$. From here on, we denote the set of cycle structures of the symmetric group $S_{m}$ by $\mathcal{C S}_{m}$. The number of permutations in $S_{m}$ with cycle structure $m^{d_{m}} \cdots 1^{d_{1}} \in \mathcal{C} \mathcal{S}_{m}$ is

$$
\frac{m !}{\prod_{i \in[m]} d_{i} ! i^{d_{i}}},
$$

as in [6, Theorem 13.2]. Further, the cycle structure of an isotopism $(\alpha, \beta, \gamma) \in \mathfrak{I}_{r, s, n}$ is defined as the triple $\left(z_{\alpha}, z_{\beta}, z_{\gamma}\right)$ formed by the respective cycle structures of $\alpha, \beta$, and $\gamma$. Keeping in mind Lemma 16 and Theorem 18, the following values are well-defined:

- $\Delta_{m}\left(z_{1}, z_{2}, z_{3}\right):=\# \operatorname{PLR}((\Theta, \mathrm{Id}) ; m)$, for any isotopism $\Theta \in \mathfrak{I}_{r, s, n}$ with cycle structure $\left(z_{1}, z_{2}, z_{3}\right)$

- $\Delta_{m}^{(12)}\left(z_{2}, z_{3}\right):=\# \operatorname{PLR}((\Theta,(12)) ; m)$, for any isotopism $\Theta=(\alpha, \beta, \gamma) \in \mathfrak{I}_{r, s, n}$ where $r=s$, such that $z_{\alpha \beta}=z_{2}$ and $z_{\gamma}=z_{3}$; and

- $\Delta_{m}^{(123)}\left(z_{3}\right):=\# \operatorname{PLR}((\Theta,(123)) ; m)$, for any isotopism $\Theta=(\alpha, \beta, \gamma) \in \mathfrak{I}_{r, s, n}$ where $r=s=n$, such that $z_{\alpha \beta \gamma}=z_{3}$.

Given a cycle structure $z \in \mathcal{C S}_{m}$, define $d_{i}^{z}:=d_{i}^{\pi}$ for any permutation $\pi \in S_{m}$ with cycle structure $z$. The next theorem follows straightforwardly from Theorem 15, Lemma 16 and (6). 
Theorem 19. Let $r, s, n \geqslant 1$ and $m \leqslant r s$. Then,

1. the number of isomorphism classes in $\operatorname{PLS}(n ; m)$ is

$$
\# \operatorname{Isom}(n ; m)=\sum_{z \in \mathcal{C} \mathcal{S}_{n}} \frac{\Delta_{m}(z, z, z)}{\prod_{i \in[n]} d_{i}^{z !} i^{d_{i}^{z}}}
$$

and

2. the number of isotopism classes in $\operatorname{PLR}(r, s, n ; m)$ is

$$
\text { \#Isot }(r, s, n ; m)=\sum_{\substack{z_{1} \in \mathcal{C S}_{r} \\ z_{\in} \in \mathcal{C} \mathcal{S}_{s} \\ z_{3} \in \mathcal{C} \mathcal{S}_{n}}} \frac{\Delta_{m}\left(z_{1}, z_{2}, z_{3}\right)}{\prod_{\substack{i \in[r] \\ j \in[s] \\ k \in[n]}} d_{i}^{z_{1}} ! d_{j}^{z_{2}} ! d_{k}^{z_{3}} ! i^{d_{1}} j^{z_{j}^{2}} k^{d_{k}^{z_{3}}}} .
$$

In practice, it is not necessary perform computations for all possible triples $\left(z_{1}, z_{2}, z_{3}\right) \in$ $\mathcal{C S}_{r} \times \mathcal{C S}_{s} \times \mathcal{C S}_{n}$ to determine the number of isotopism classes in statement 2 of Theorem 19. The following lemma gives necessary and sufficient conditions for $\operatorname{PLR}((\Theta, I d))$ to contain a non-empty partial Latin rectangle. This generalizes in a natural way a pair of similar results concerning Latin squares [64, Lemma 3.6] and partial Latin squares [27, Lemma 2.2].

Lemma 20. Let $\Theta \in \mathfrak{I}_{r, s, n}$ be an isotopism of cycle structure $\left(z_{1}, z_{2}, z_{3}\right) \in \mathcal{C} \mathcal{S}_{r} \times \mathcal{C} \mathcal{S}_{s} \times \mathcal{C S}_{n}$. The set $\operatorname{PLR}((\Theta, \mathrm{Id}))$ contains at least one non-empty partial Latin rectangle if and only if there exists a triple $(i, j, k) \in[r] \times[s] \times[n]$ such that the following two conditions are satisfied:

1. $\operatorname{lcm}(i, j)=\operatorname{lcm}(i, k)=\operatorname{lcm}(j, k)=\operatorname{lcm}(i, j, k)$, and

2. $z_{1}$ has an $i$-cycle, $z_{2}$ has a $j$-cycle, and $z_{3}$ has a $k$-cycle.

Proof. Let us suppose that $\Theta=(\alpha, \beta, \gamma)$. If $(u, v, w) \in[r] \times[s] \times[n]$ is an entry of a non-empty partial Latin rectangle $L \in \operatorname{PLR}((\Theta, \mathrm{Id}))$, then let $\alpha^{\prime}, \beta^{\prime}$ and $\gamma^{\prime}$ be the respective cycles in the unique decompositions of the permutations $\alpha, \beta$ and $\gamma$ such that $\alpha(u)=\alpha^{\prime}(u), \beta(v)=\beta^{\prime}(v)$ and $\gamma(w)=\gamma^{\prime}(w)$. Since $\Theta$ is an autotopism of $L$, Conditions (1) and (2) hold for the triple $(i, j, k) \in[r] \times[s] \times[n]$ that is formed by the respective lengths of the cycles $\alpha^{\prime}, \beta^{\prime}$ and $\gamma^{\prime}$.

Reciprocally, if Condition (2) holds, then there exist a triple $(u, v, w) \in[r] \times[s] \times[n]$, an $i$-cycle $\alpha^{\prime}$ of $\alpha$, a $j$-cycle $\beta^{\prime}$ of $\beta$ and a $k$-cycle $\gamma^{\prime}$ of $\gamma$ such that $\alpha(u)=\alpha^{\prime}(u), \beta(v)=\beta^{\prime}(v)$ and $\gamma(w)=\gamma^{\prime}(w)$. Then, Condition (1) implies that $\Theta$ is an autotopism of the partial Latin rectangle $L \in \operatorname{PLR}(r, s, n)$ that is described so that

$$
E(L)=\left\{\left(\alpha^{\prime m}(u), \beta^{\prime m}(v), \gamma^{\prime m}(w)\right): 0 \leqslant m<\operatorname{lcm}(i, j, k)\right\} .
$$


Parastrophisms preserve the number of isotopism and main classes of partial Latin rectangles of a given order. Thus, in practice, it is enough to focus on the case $r \leqslant s \leqslant n$ to determine the number of isotopism classes in $\operatorname{PLR}(r, s, n ; m)$, whereas the number of main classes splits into three cases: (a) $r<s<n$; (b) $r=s<n$; and (c) $r=s=n$. Notice that, from parastrophism, the case $r<s=n$ follows similarly to (b), because the important aspect in that case is that exactly two dimensions of the partial Latin rectangle under consideration coincide. Now, in (a), the parastrophism group $S_{r, s, n}$ is only formed by the trivial permutation Id $\in S_{3}$ and hence, the number of main classes coincides with that of isotopism classes. In order to deal with (b) and (c), and keeping in mind Theorem 18, let us define the following two sets for each pair of permutations $\beta, \gamma \in S_{r}$.

$$
\begin{gathered}
C_{1}(\beta, \gamma):=\left\{\left(\alpha, \beta^{\prime}, \gamma^{\prime}\right) \in \mathfrak{I}_{r, r, n}: \alpha \beta^{\prime} \sim \beta \text { and } \gamma^{\prime} \sim \gamma\right\} . \\
C_{2}(\gamma):=\left\{\left(\alpha, \beta, \gamma^{\prime}\right) \in \mathfrak{I}_{r, r, r}: \alpha \beta \gamma^{\prime} \sim \gamma\right\} .
\end{gathered}
$$

The next result holds straightforwardly from Theorem 15, Lemma 16, and (6).

Theorem 21. Let $r, n \geqslant 1$ and $m \leqslant r^{2}$. The following statements hold.

1. If $n \neq r$, then the number of main classes in $\operatorname{PLR}(r, r, n ; m)$ is

$$
\# \mathrm{MC}(r, r, n ; m)=\frac{\# \operatorname{Isot}(r, r, n ; m)}{2}+\frac{1}{2 r !^{2} n !} \sum_{(\beta, \gamma) \in S_{s} \times S_{n}}\left|C_{1}(\beta, \gamma)\right| \Delta_{m}^{(12)}\left(z_{\beta}, z_{\gamma}\right) .
$$

2. The number of main classes in $\operatorname{PLR}(r, r, r ; m)$ is

$$
\begin{aligned}
\# \mathrm{MC}(r, r, r ; m)= & \frac{\# \operatorname{Isot}(r, r, r ; m)}{6}+\frac{1}{2 r !^{3}} \sum_{(\beta, \gamma) \in S_{s} \times S_{n}}\left|C_{1}(\beta, \gamma)\right| \Delta_{m}^{(12)}\left(z_{\beta}, z_{\gamma}\right) \\
& +\frac{1}{3 r !^{3}} \sum_{\gamma \in S_{n}}\left|C_{2}(\gamma)\right| \Delta_{m}^{(123)}\left(z_{\gamma}\right) .
\end{aligned}
$$

\section{Computational results}

In this section, we enumerate all the computational results that we have obtained from the implementation of the various proposed methods. All of them are shown in Tables 2-15, which are listed in Appendix B. Let us provide here a guide to these tables by describing the existing relationship with each one of the proposed methods.

Tables 2-3: Graphs and polynomials for computing $f_{m}(r, s, n)$ For small graphs $G$, Tables 2 and 3 list the polynomial $P(G)=P(G ; r, s, n)$ that is necessary for dealing with the inclusion-exclusion method described in Theorem 6 . These polynomials were computed using a $\mathrm{C}++$ program, using geng (packaged with nauty $[48,49,51]$ ) to generate a list of isolated-vertex-free non-isomorphic graphs (e.g. "geng - d1 3" generates 
3-vertex isomorphism class representatives with minimum degree 1) and bliss [43] to compute their automorphism group size. The notation $\overline{a b c}$ is shorthand for the sum of the monic monomials with variables $r, s$, and $n$ and exponents $a, b$, and $c$. For example, $\overline{210}=r^{2} s+r^{2} n+s^{2} r+s^{2} n+n^{2} r+n^{2} s$ and $2 \overline{100}=2(r+s+n)$.

By Lemma 9, substituting the data in Tables 2 and 3 into the formula in Theorem 6 gives a formula for $f_{m}(r, s, n)$ containing all terms of degree $\geqslant 3 m-9$; unlisted graphs $G$ have $v-c(G) \geqslant 5$, and thus contribute to terms in the polynomial with degree at most $3 m-10$. In this regard, the following result generalizes Theorem 4.7 in [30], which only deals with the case $m \leqslant 6$.

Theorem 22. Let $m$ be a positive integer. Then,

$$
\begin{aligned}
& f_{m}(r, s, n)= \\
& \left(\begin{array}{c}
m \\
2
\end{array}\right)(r s n)^{m-1}(2-\overline{100}) \\
& \left(\begin{array}{c}
m \\
3
\end{array}\right)(r s n)^{m-2}(14-12 \overline{100}+6 \overline{110}+2 \overline{200}) \\
& +\quad\left(\begin{array}{c}
m \\
4
\end{array}\right)(r s n)^{m-3}(198-228 \overline{100}+198 \overline{110}-84 \overline{111}+72 \overline{200}-36 \overline{210} \\
& -12 \overline{211}+6 \overline{221}-6 \overline{300}+3 \overline{311}) \\
& +\left(\begin{array}{c}
m \\
5
\end{array}\right)(r s n)^{m-4}(-6360 \overline{100}+7440 \overline{110}-6080 \overline{111}+2880 \overline{200}-2520 \overline{210} \\
& +820 \overline{211}+480 \overline{220}+360 \overline{221}-180 \overline{222}-480 \overline{300}+240 \overline{310} \\
& +160 \overline{311}-80 \overline{321}+24 \overline{400}-20 \overline{411}) \\
& +\quad\left(\begin{array}{c}
m \\
6
\end{array}\right)(\text { rsn })^{m-5}(-13170 \overline{211}+17340 \overline{221}-15990 \overline{222}+7580 \overline{311} \\
& -7050 \overline{321}+3300 \overline{322}+1520 \overline{331}+180 \overline{332}-90 \overline{333}-1740 \overline{411} \\
& +870 \overline{421}+90 \overline{422}-45 \overline{432}+130 \overline{511}-15 \overline{522}) \\
& +\quad\left(\begin{array}{c}
m \\
7
\end{array}\right)(r s n)^{m-6}(-10920 \overline{322}+15540 \overline{332}-15120 \overline{333}+7350 \overline{422} \\
& -7140 \overline{432}+3570 \overline{433}+1680 \overline{442}-2100 \overline{522}+1050 \overline{532}+210 \overline{622}) \\
& +\left(\begin{array}{c}
m \\
8
\end{array}\right)(r s n)^{m-7}(-3360 \overline{433}+5040 \overline{443}-5040 \overline{444}+2520 \overline{533}-2520 \overline{543} \\
& +1260 \overline{544}+630 \overline{553}-840 \overline{633}+420 \overline{643}+105 \overline{733}) \\
& + \\
& \text { some polynomial of degree } \leqslant 3 m-10 \text {. }
\end{aligned}
$$

Theorem 22 is exact for $m \leqslant 5$ since Tables 2 and 3 contain all graphs with no isolated vertices with up to 5 vertices, and graphs with $v \geqslant 6$ make a zero contribution to the expression for $f_{m}(r, s, n)$ in Theorem 6 since the binomial $\left(\begin{array}{c}m \\ v\end{array}\right)=0$. Furthermore, we have made use of the chromatic polynomial method described in Theorem 10 to compute exact 
formulas for $f_{m}(r, s, n)$ for all $m \leqslant 13$ which are available from [35]. They corroborate in particular the formulas shown in [30] for $m \leqslant 6$. The authors acknowledge the use of GAP [38], the GAP package GRAPE [60] (which uses nauty), and the Tutte polynomial software tutte_bhkk [12] (available from github.com/thorehusfeldt/tutte_bhkk) for these computations.

Tables 4-8: Number of partial Latin rectangles We have implemented Sade's method described in Algorithm 1 in $\mathrm{C}++$ using nauty for graph isomorphism and GMP [40] for arbitrary precision arithmetic, which we use to compute the value \#PLR $(r, s, n ; m)$ for all $r, s, n \leqslant 7$, and for $r, s \leqslant 6$ when $n=8$ (for all $0 \leqslant m \leqslant r s$ ). Our computations for $r, s, n \leqslant 6$ corroborate Tables 2 through 5 in [30]. The remaining cases are listed here in Tables 4 through 8 .

Tables 9-15: Number of isomorphism, isotopism and main classes We have implemented the algebraic geometry method described in Theorem 12 in both Singular [19] and Minion [39] to determine the values $\Delta\left(z_{1}, z_{2}, z_{3}\right)$, for all $\left(z_{1}, z_{2}, z_{3}\right) \in \mathcal{C} \mathcal{S}_{r} \times \mathcal{C S}_{s} \times$ $\mathcal{C S}_{n}$ satisfying the conditions of Lemma 20, when $r, s, n \leqslant 6$. Theorem 19 has then be applied to obtain the corresponding numbers of isomorphism and isotopism classes of partial Latin rectangles, as listed in Tables 9 through 12.

The number of main classes of partial Latin rectangles in $\operatorname{PLR}(r, s, n)$ according to their weights is given in Tables 13 and 14 when $2 \leqslant r \leqslant s \leqslant n \leqslant 6$. We include only the cases in which $r, s$, and $n$ are not pairwise distinct; otherwise, the number of main classes and isotopism classes coincide.

Furthermore, it is also possible to enumerate constructively the number of isotopism and main classes in the set $\operatorname{PLR}(r, s, n ; m)$. We simply extend all representative weight$(m-1)$ partial Latin rectangles by one entry in all possible ways, and throw away those that belong to the same class as an already discovered partial Latin rectangle. To compare isotopism and main class equivalence, it is enough, for instance, to generate a graph similar to those proposed in [50].

For fixed $m \geqslant 1$, provided $r \geqslant m, s \geqslant m$, and $n \geqslant m$, the number of isotopism classes and main classes in the set $\operatorname{PLR}(r, s, n ; m)$ do not vary with $r, s$ and $n$, which amounts to adding empty rows, empty columns, or unused symbols. To compute these numbers, we use the above constructive method, but allow the possibility of introducing new rows, columns, and/or symbols when extending weight- $(m-1)$ partial Latin rectangles. We perform this enumeration for $m \leqslant 11$, and the results are given in Table 15 . The results for main classes is consistent with those independently obtained in [20,73], and moreover, [20] also computes the number of main classes for $m=12$.

Direct constructive enumeration of isomorphism classes is infeasible, since the numbers grow too quickly. Moreover, isotopic partial Latin rectangles may have different-sized isomorphism classes. In the context of Latin squares, this led to [50, Th. 2(i)] being false; which is acknowledged in a corrected version of [50] on McKay's website

http://users.cecs.anu.edu.au/ bdm/papers/ls_final_corr.pdf. 
Thus, we cannot easily derive the number of isomorphism classes within a isotopism class, which thwarts modifying the approach we use for enumerating isotopism classes to enumerating isomorphism classes. Instead, using an algebraic geometry method like in Section 5, we enumerate isomorphism classes for $m \leqslant 6$ in [34, Table 2].

\section{Verification}

The authors have made efforts to ensure the numbers and formulas presented here are as bug-free as possible; we document these efforts in this section. First, notice that some results can immediately be checked by taking into account the already known results mentioned in the introductory section, particularly those ones concerning Latin rectangles $[41,50,53,61,62,70]$ and partial Latin rectangles of small orders $[1,20,27,28,30,34$, 73]. Further, the various source codes used and their output are available from [35]. Next, where feasible, computations have been independently performed, using different techniques and different software. Where possible, we have also cross-checked the results of the enumeration methods.

- The computation of \#PLR $(r, s, n ; m)$ for all $r, s, n \leqslant 7$ has been performed using both the algebraic geometry method (except for $(s, n) \in\{(6,7),(7,7)\}$ ) and Sade's method.

- The number of isotopism classes and main classes has been computed using both the algebraic geometry method (for $r, s, n \leqslant 6$ ) and constructive enumeration (for $r, s, n \leqslant 5)$.

- For $m \leqslant 13$, the results of the computation of \#PLR $(r, s, n ; m)$ have been crosschecked against the computed polynomials $f_{m}(r, s, n)$. Thus for 854 quadruples $(r, s, n ; m)$ the computations agreed exactly.

In addition to cross-checking computational results, we check the divisibility of the numbers computed using the following theorem. More specifically, we check that the exact formulas for $f_{m}(r, s, n)$, for $m \leqslant 13$, satisfy Theorem 23 whenever $k \in\{1, \ldots, 10\}$ and $r, s, n \in\{k+1, \ldots, k+10\}$.

Theorem 23. For all $r, s, n, m \geqslant 1$ and $k \geqslant 0$, we have

$$
\begin{aligned}
& \# \operatorname{PLR}(r, s, n ; m) \equiv \# \operatorname{PLR}(k, s, n ; m) \quad(\bmod r-k) \text { when } r \geqslant k+1, \\
& \# \operatorname{PLR}(r, s, n ; m) \equiv \# \operatorname{PLR}(r, k, n ; m) \quad(\bmod s-k) \text { when } s \geqslant k+1, \text { and } \\
& \# \operatorname{PLR}(r, s, n ; m) \equiv \# \operatorname{PLR}(r, s, k ; m) \quad(\bmod n-k) \text { when } n \geqslant k+1 .
\end{aligned}
$$

Proof. Firstly, we prove the second claim. We act on $\operatorname{PLR}(r, s, n ; m)$ by permuting the columns using the group $G$ of isotopisms generated by (Id, $\beta, \operatorname{Id})$ for an $(m-k)$-cycle $\beta$. By the Orbit-Stabilizer Theorem, orbits have size $m-k$ unless they contain partial Latin rectangles that admit a non-trivial autotopism in $G$. This is only possible if the columns permuted by $\beta$ are empty. Hence, the orbits of size less than $m-k$ together form $\operatorname{PLR}(r, k, n ; m)$ by deleting the columns permuted by $\beta$. The same argument works for rows and symbols, which gives the first and third claims. 


\section{Acknowledgements}

The authors want to express their gratitude to the anonymous referees for the comprehensive reading of the paper and their pertinent comments and suggestions, which helped improve the manuscript. Stones would also like to acknowledge the use of the website math.stackexchange.com for discussing problems arising in this work. Thanks to Zhuanhao $\mathrm{Wu}$ for parallelizing and improving the memory management in Stones's $\mathrm{C}++$ code. Thanks also to Daniel Kotlar for assistance in computing autotopism groups, which is leading to the papers $[17,18,69]$.

\section{References}

[1] P. Adams, R. Bean, and A. Khodkar, A census of critical sets in the Latin squares of order at most six, Ars Comb. 68 (2003), 203-223.

[2] W. Adams and P. Loustaunau, An introduction to Gröbner bases, Graduate Studies in Mathematics 3, Am. Math. Soc., 1994.

[3] S. D. Andres, H. Bergold, and R. M. Falcón, Autoparatopism stabilized colouring games on rook's graphs, Electron. Notes Discret. Math. 68 (2018), 233-238.

[4] S. D. Andres and R. M. Falcón, Autotopism stabilized colouring games on rook's graphs, Discrete Appl. Math. 266 (2019), 200-212.

[5] S. D. Andres and R. M. Falcón, Colouring games based on autotopisms of Latin hyper-rectangles, Quaest. Math. 42 (2019), 953-975.

[6] G. E. Andrews, The theory of partitions, Cambridge University Press, 1984.

[7] E. Arnold, S. Lucas, and L. Taalman, Gröbner basis representations of sudoku, College Mathematics Journal 41 (2010), 101-112.

[8] R. Artzy, A note on the automorphisms of special loops, Riveon Lematematika 8 (1954), 81, In Hebrew.

[9] R. A. Bailey, Latin squares with highly transitive automorphism groups, J. Aust. Math. Soc. 33 (1982), 18-22.

[10] S. E. Bammel and J. Rothstein, The number of $9 \times 9$ Latin squares, Discrete Math. 11 (1975), 93-95.

[11] D. Bayer, The division algorithm and the Hilbert scheme, Ph.D. Thesis, Harvard University, 1982.

[12] A. Björklund, T. Husfeldt, P. Kaski, and M. Koivisto, Computing the Tutte polynomial in vertex-exponential time, Proc. IEEE Foundations of Computer Science, 2008, pp. 677-686.

[13] J. Browning, D. S. Stones, and I. M. Wanless, Bounds on the number of autotopisms and subsquares of a Latin square, Combinatorica 33 (2013), 11-22.

[14] D. Bryant, M. Buchanan, and I. M. Wanless, The spectrum for quasigroups with cyclic automorphisms and additional symmetries, Discrete Math. 304 (2009), 821-833. 
[15] N. J. Cavenagh and D. S. Stones, Near-automorphisms of Latin squares, J. Combin. Des. 19 (2011), 365-377.

[16] D. A. Cox, J. B. Little, and D. O'Shea, Ideals, varieties, and algorithms. an introduction to computational algebraic geometry and commutative algebra, Springer, New York, 2007.

[17] E. Danan, R. M. Falcón, D. Kotlar, T. G. Marbach, and R. J. Stones, Two-line graphs of partial Latin rectangles, Electron. Notes Discret. Math. 68 (2018), 53-58.

[18] E. Danan, R. M. Falcón, D. Kotlar, T. G. Marbach, and R. J. Stones, Refining invariants for computing autotopism groups of partial Latin rectangles, Discrete Math. 343 (2020), paper 111812.

[19] W. Decker, G.-M. Greuel, G. Pfister, and H. Schönemann, Singular 4-1-2 - A computer algebra system for polynomial computations, 2019, http://www. singular.uni-kl.de.

[20] H. Dietrich and I. M. Wanless, Small partial Latin squares that embed in an infinite group but not into any finite group, J. Symbolic Comput. 86 (2018), 142-152.

[21] A. A. Drisko, Loops of order $p^{n}+1$ with transitive automorphism groups, Adv. Math. 128 (1997), 36-39.

[22] O. J. Falcón, R. M. Falcón, J. Núñez, A. M. Pacheco, and M. T. Villar, Computation of isotopisms of algebras over finite fields by means of graph invariants, J. Comput. Appl. Math 318 (2017), 307-315.

[23] R. M. Falcón, Latin squares associated to principal autotopisms of long cycles. Application in cryptography, Proc. Transgressive Computing, 2006, pp. 213-230.

[24] R. M. Falcón, Study of critical sets in Latin squares by using the autotopism group, Electron. Notes Discret. Math. 29 (2007), 503-507.

[25] R. M. Falcón, 0/1-polytopes related to Latin squares autotopisms, Proc. VI Jornadas de Matemática Discreta y Algorítmica, 2008, pp. 311-319.

[26] R. M. Falcón, Cycle structures of autotopisms of the Latin squares of order up to 11, Ars Combin. 103 (2012), 239-256.

[27] R. M. Falcón, The set of autotopisms of partial Latin squares, Discrete Math. 313 (2013), 1150-1161.

[28] R. M. Falcón, Enumeration and classification of self-orthogonal partial Latin rectangles by using the polynomial method, European J. Combin. 48 (2015), 215-223.

[29] R. M. Falcón, Using a CAS/DGS to analyze computationally the configuration of planar bar linkage mechanisms based on partial Latin squares, Math. Comput. Sci. 14 (2020), 375-389.

[30] R. M. Falcón, O. J. Falcón, and J. Núñez, Counting and enumerating partial Latin rectangles by means of computer algebra systems and CSP solvers, Math. Methods Appl. Sci. 41 (2018), 7236-7262.

[31] R. M. Falcón, O. J. Falcón, and J. Núñez, A historical perspective of the theory of isotopisms, Symmetry 10 (2018), paper 322. 
[32] R. M. Falcón and J. Martín-Morales, Gröbner bases and the number of Latin squares related to autotopisms of order $\leqslant 7$, J. Symbolic Comput. 42 (2007), 1142-1154.

[33] R. M. Falcón and J. Núñez, Partial Latin squares having a Santilli's autotopism in their autotopism groups, J. Dyn. Syst. Geom. Theor. 5 (2007), 19-32.

[34] R. M. Falcón and R. J. Stones, Classifying partial Latin rectangles, Electronic Notes in Discrete Mathematics 49 (2015), 765-771.

[35] R. M. Falcón and R. J. Stones, Enumerating partial Latin rectangles, Mendeley Data, v1 (2017), http://dx.doi.org/10.17632/4c559g2xny.1.

[36] R. M. Falcón and R. J. Stones, Partial Latin rectangle graphs and autoparatopism groups of partial Latin rectangles with trivial autotopism groups, Discrete Math. 340 (2017), 1242-1260.

[37] J. Gago-Vargas, I. Hartillo-Hermoso, J. Martín-Morales, and J. M. Ucha-Enríquez, Sudokus and Gröbner bases not only a divertimento, Lect. Notes Comput. Sc. 4194 (2006), 155-165.

[38] The GAP Group, GAP - Groups, algorithms, programming - A system for computational discrete algebra, (2017), http://www.gap-system.org/.

[39] I. P. Gent, C. Jefferson, and I. Miguel, Minion: a fast scalable constraint solver, Proc. European Conference on Artificial Intelligence (G. Brewka, S. Coradeschi, A. Perini, and P. Traverso., eds.), 2006, pp. 98-102.

[40] T. Granlund et al., GNU multiple precision arithmetic library, (2017), http:// gmplib.org/.

[41] A. Hulpke, P. Kaski, and P. R. J. Östergård, The number of Latin squares of order 11, Math. Comp. 80 (2011), 1197-1219.

[42] E. C. Ihrig and B. M. Ihrig, The recognition of symmetric Latin squares, J. Combin. Des. 16 (2008), 291-300.

[43] T. Junttila and P. Kaski, Engineering an efficient canonical labeling tool for large and sparse graphs, Proc. Workshop on Algorithm Engineering and Experiments and the Workshop on Analytic Algorithms and Combinatorics, 2007, pp. 135-149.

[44] B. Kerby and J. D. H. Smith, Quasigroup automorphisms and symmetric group characters, Comment. Math. Univ. Carol. 51 (2010), 279-286.

[45] B. Kerby and J. D. H. Smith, Quasigroup automorphisms and the Norton-Stein complex, Proc. Amer. Math. Soc. 138 (2010), 3079-3088.

[46] M. Kreuzer and L. Robbiano, Computational commutative algebra, Springer-Verlag Berlin Heidelberg, 2000.

[47] C. F. Laywine and G. L. Mullen, Discrete Mathematics using Latin squares, Series in Discrete Mathematics and Optimization, Wiley-Interscience, 1998.

[48] B. D. McKay, Practical graph isomorphism, Congr. Numer. 30 (1981), 45-87.

[49] B. D. McKay, NAUTY - Graph isomorphic software, (2017), http://cs.anu.edu.au/ bdm/nauty/. 
[50] B. D. McKay, A. Meynert, and W. Myrvold, Small Latin squares, quasigroups, and loops, J. Combin. Des. 15 (2007), 98-119.

[51] B. D. McKay and A. Piperno, Practical graph isomorphism, II, J. Symbolic Computation 60 (2014), 94-112.

[52] B. D. McKay and E. Rogoyski, Latin squares of order ten, Electron. J. Combin. 2 (1995), \#N3.

[53] B. D. McKay and I. M. Wanless, On the number of Latin squares, Ann. Comb. 9 (2005), 335-344.

[54] B. D. McKay, I. M. Wanless, and X. Zhang, The order of automorphisms of quasigroups, J. Combin. Des. 23 (2015), 275-288.

[55] M. J. L. Mendis and I. M. Wanless, Latin squares with a unique intercalate, J. Combin. Des. 24 (2016), 79-293.

[56] M. J. L. Mendis and I. M. Wanless, Autoparatopisms of quasigroups and Latin squares, J. Combin. Des. 25 (2017), 51-74.

[57] H. Meng, Y. Zheng, and Y. Zheng, The classification construction and the nonisomorphism counting of symmetric Latin square, Adv. Stud. Contemp. Math. (Kyungshang) 17 (2008), 169-179.

[58] A. A. Sade, Énumération des Carrés Latins, Application au $7^{e}$ Ordre, Conjecture pour les Ordres Supérieurs, privately published (1948), 8 pp.

[59] Y. Sato, S. Inoue, A. Suzuki, K. Nabeshi, and K. Sakai, Boolean Gröbner bases, J. Sym. Comp. 46 (2011), 622-632.

[60] L. H. Soicher, GRAPE - A GAP package for computing with graphs and groups, (2017), http://www.maths.qmul.ac.uk/ leonard/grape/.

[61] D. S. Stones, The many formulae for the number of Latin rectangles, Electron. J. Combin. 17 (2010), \#A1.

[62] D. S. Stones, On the number of Latin rectangles, Ph.D. thesis, Monash University, 2010, http://arrow.monash.edu.au/hdl/1959.1/167114.

[63] D. S. Stones, Symmetries of partial Latin squares, European J. Combin. 34 (2013), 1092-1107.

[64] D. S. Stones, P. Vojtěchovský, and I. M. Wanless, Cycle structure of autotopisms of quasigroups and Latin squares, J. Combin. Des. 20 (2012), 227-263.

[65] D. S. Stones and I. M. Wanless, Divisors of the number of Latin rectangles, J. Combin. Theory Ser. A 117 (2010), 204-215.

[66] D. S. Stones and I. M. Wanless, A congruence connecting Latin rectangles and partial orthomorphisms, Ann. Comb. 16 (2012), 349-365.

[67] D. S. Stones and I. M. Wanless, How not to prove the Alon-Tarsi Conjecture, Nagoya Math. J. 205 (2012), 1-24. 
[68] R. J. Stones, K-plex 2-erasure codes and blackburn partial Latin squares, IEEE Trans. Inf. Theory Early access (2020), 1-1, https://doi.org/10.1109/TIT.2020.2967758.

[69] R. J. Stones, R. M. Falcón, D. Kotlar, and T. G. Marbach, Computing autotopism groups of partial latin rectangles: a pilot study, Comput. Math. Method. Early view (2020), paper e1094, https://doi.org/10.1002/cmm4.1094.

[70] R. J. Stones, S. Lin, X. Liu, and G. Wang, On computing the number of Latin rectangles, Graphs. Combin. 32 (2016), 1187-1202.

[71] R. J. Stones, M. Su, X. Liu, G. Wang, and S. Lin, A Latin square autotopism secret sharing scheme, Des. Codes Cryptogr. 35 (2015), 1-16.

[72] I. M. Wanless and E. C. Ihrig, Symmetries that Latin squares inherit from 1factorizations, J. Combin. Des. 13 (2005), 157-172.

[73] I. M. Wanless and B. S. Webb, Small partial Latin squares that cannot be embedded in a Cayley table, Australas. J. Combin. 67 (2017), 352-363.

[74] M. B. Wells, The number of Latin squares of order eight, J. Combin. Theory 3 (1967), 98-99.

[75] M. Yan, J. Feng, T. G. Marbach, R. J. Stones, G. Wang, and X. Liu, Gecko: A resilient dispersal scheme for multi-cloud storage, IEEE Access 7 (2019), 7738777397.

[76] L. Yi, R. J. Stones, and G. Wang, Two-erasure codes from 3-plexes, Network and Parallel Computing (Cham) (X. Tang, Q. Chen, P. Bose, W. Zheng, and J.-L. Gaudiot, eds.), Springer International Publishing, 2019, pp. 264-276.

\section{A Glossary of symbols}

$\begin{array}{ll}S_{n} & \text { The symmetric group on } n \text { elements. } \\ \mathfrak{I}_{r, s, n} & \text { The isotopism group } S_{r} \times S_{s} \times S_{n} . \\ S_{r, s, n} & \text { The parastrophism group defined in Section } 1 . \\ \mathfrak{P}_{r, s, n} & \text { The paratopism group } S_{r} \times S_{s} \times S_{n} \rtimes S_{r, s, n} . \\ \operatorname{PLR}(r, s, n) & \text { The set of } r \times s \text { partial Latin rectangles on }[n] \cup\{\cdot\} . \\ \operatorname{PLR}(r, s, n ; m) & \text { The subset of partial Latin rectangles in } \operatorname{PLR}(r, s, n) \text { of weight } m . \\ \operatorname{PLR}((\Theta, \pi)) & \text { The set of partial Latin rectangles having }(\Theta, \pi) \text { as autoparatopism. } \\ \operatorname{PLR}((\Theta, \pi) ; m) & \text { The subset of partial Latin rectangles in } \operatorname{PLR}((\Theta, \pi)) \text { of weight } m . \\ \operatorname{PLS}(n ; m) & \text { The set of partial Latin squares of order } n \text { and weight } m . \\ \operatorname{Isom}(n ; m) & \text { The set of isomorphism classes of } \operatorname{PLS}(n ; m) . \\ \operatorname{Isot}(r, s, n ; m) & \text { The set of isotopism classes of } \operatorname{PLR}(r, s, n ; m) . \\ \operatorname{MC}(r, s, n ; m) & \text { The set of main classes of } \operatorname{PLR}(r, s, n ; m) .\end{array}$




\section{B Tables}

\begin{tabular}{|c|c|c|c|}
\hline block $K$ & induced subgraph & $\left|\operatorname{Aut}\left(G_{K}\right)\right|$ & $\Pi(K ; n)$ \\
\hline 1 & $\bullet$ & 1 & $n$ \\
\hline $\begin{array}{|ll|}1 & 1 \\
\end{array}$ & $\bullet \bullet$ & 2 & $n(n-1)$ \\
\hline $\begin{array}{|lll|}1 & 1 & 1 \\
\end{array}$ & $\bullet$ & 6 & $n(n-1)(n-2)$ \\
\hline $\begin{array}{|ll|}1 & 1 \\
1 & 0 \\
\end{array}$ & & 1 & $n(n-1)^{2}$ \\
\hline $\begin{array}{llll}1 & 1 & 1 & 1 \\
\end{array}$ & $\cdots$ & 24 & $n(n-1)(n-2)(n-3)$ \\
\hline $\begin{array}{|lll|}1 & 1 & 1 \\
1 & 0 & 0 \\
\end{array}$ & $\because \bullet$ & 2 & $n(n-1)^{2}(n-2)$ \\
\hline $\begin{array}{|lll|}1 & 1 & 0 \\
1 & 0 & 1 \\
\end{array}$ & & 2 & $n(n-1)^{3}$ \\
\hline $\begin{array}{|ll|}1 & 1 \\
1 & 1 \\
\end{array}$ & & 4 & $n(n-1)\left(n^{2}-3 n+3\right)$ \\
\hline $\begin{array}{llll}1 & 1 & 1 & 1 \\
\end{array}$ & $\infty 0$ & 120 & $n(n-1)(n-2)(n-3)(n-4)$ \\
\hline $\begin{array}{|llll|}1 & 1 & 1 & 1 \\
1 & 0 & 0 & 0\end{array}$ & $\because \infty$ & 6 & $n(n-1)^{2}(n-2)(n-3)$ \\
\hline \begin{tabular}{|llll}
1 & 1 & 1 & 0 \\
1 & 0 & 0 & 1 \\
\end{tabular} & $\because \bullet$ & 2 & $n(n-1)^{3}(n-2)$ \\
\hline $\begin{array}{|lll|}1 & 1 & 1 \\
1 & 1 & 0 \\
\end{array}$ & & 2 & $n(n-1)(n-2)\left(n^{2}-3 n+3\right)$ \\
\hline $\begin{array}{|lll|}1 & 1 & 1 \\
1 & 0 & 0 \\
1 & 0 & 0 \\
\end{array}$ & $0^{\circ}$ & 4 & $n(n-1)^{2}(n-2)^{2}$ \\
\hline $\begin{array}{|lll|}1 & 1 & 1 \\
1 & 0 & 0 \\
0 & 1 & 0 \\
\end{array}$ & $\because ?$ & 2 & $n(n-1)^{3}(n-2)$ \\
\hline $\begin{array}{lll}1 & 1 & 0 \\
1 & 0 & 1 \\
1 & 0 & 0 \\
\end{array}$ & & 2 & $n(n-1)^{3}(n-2)$ \\
\hline $\begin{array}{lll}1 & 1 & 0 \\
1 & 0 & 1 \\
0 & 1 & 0\end{array}$ & 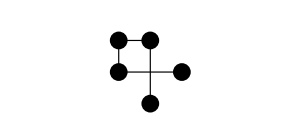 & 1 & $n(n-1)^{4}$ \\
\hline
\end{tabular}

Table 1: The blocks $K$ with $\leqslant 5$ ones, along with $\left|\operatorname{Aut}\left(G_{K}\right)\right|$ and its chromatic polynomial $\Pi(K ; n)$. 


\begin{tabular}{|c|c|c|c|c|c|}
\hline$G$ & $v$ & $e$ & $c(G)$ & $|\operatorname{Aut}(G)|$ & $P(G)=P(G ; r, s, n)$ \\
\hline$\bullet$ & 2 & 1 & 1 & 2 & $\overline{100}-2$ \\
\hline ob & 3 & 2 & 1 & 2 & $P(\bullet)^{2}$ \\
\hline A. & 3 & 3 & 1 & 6 & $\overline{200}-2$ \\
\hline 88 & 4 & 2 & 2 & 8 & $\overline{111} P(\bullet)^{2}$ \\
\hline 8 & 4 & 3 & 1 & 6 & $P(\bullet)^{3}$ \\
\hline & 4 & 3 & 1 & 2 & $P(\bullet)^{3}$ \\
\hline $\mathbb{S}^{\circ}$ & 4 & 4 & 1 & 2 & $P(\boldsymbol{\curvearrowright}) P(\bullet)$ \\
\hline 20 & 4 & 4 & 1 & 8 & $\overline{300}+6 \overline{110}-12 \overline{100}+16$ \\
\hline 28 & 4 & 5 & 1 & 4 & $\overline{300}+2 \overline{110}-4 \overline{100}+4$ \\
\hline 88 & 4 & 6 & 1 & 24 & $\overline{300}-2$ \\
\hline 8. & 5 & 3 & 2 & 4 & $\overline{111} P(\bullet \bullet)^{3}$ \\
\hline AB & 5 & 4 & 2 & 12 & $\overline{111} P(\boldsymbol{\Delta}) P(\bullet \bullet)$ \\
\hline & 6 & 3 & 3 & 48 & $\overline{222} P(\bullet \bullet)^{3}$ \\
\hline
\end{tabular}

Table 2: The polynomial $P(G)$ for $v$-vertex graphs $G$ with $v-c(G) \leqslant 3$. 


\begin{tabular}{|c|c|c|c|c|c|}
\hline$G$ & $v$ & $e$ & $c(G)$ & $|\operatorname{Aut}(G)|$ & $P(G)=P(G ; r, s, n)$ \\
\hline 28 & 5 & 4 & 1 & 24 & $P(\bullet \bullet)^{4}$ \\
\hline 82. & 5 & 4 & 1 & 2 & $P(\bullet)^{4}$ \\
\hline 80 & 5 & 5 & 1 & 4 & $P(\boldsymbol{\bullet}) P(\bullet)^{2}$ \\
\hline 20 & 5 & 5 & 1 & 2 & $P(\mathfrak{\bullet}) P(\bullet)$ \\
\hline 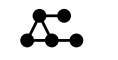 & 5 & 5 & 1 & 2 & $P(\boldsymbol{\bullet}) P(\bullet)^{2}$ \\
\hline 8 & 5 & 6 & 1 & 2 & $P(\not{20}) P(\bullet \bullet)$ \\
\hline$\infty$ & 5 & 6 & 1 & 12 & $P(\mathscr{\bullet}) P(\bullet)$ \\
\hline$\infty$ & 5 & 7 & 1 & 12 & $\overline{400}+3 \overline{210}+6 \overline{111}+3 \overline{211}-6 \overline{200}-12 \overline{110}+12 \overline{100}-8$ \\
\hline 886 & 5 & 4 & 1 & 2 & $P(\bullet)^{4}$ \\
\hline & 5 & 5 & 1 & 2 & $P(\boldsymbol{\triangleleft}) P(\bullet \bullet)^{2}$ \\
\hline 88 & 5 & 6 & 1 & 8 & $P(\mathbf{A})^{2}$ \\
\hline & 5 & 5 & 1 & 10 & $\overline{400}+10 \overline{210}-20 \overline{200}-30 \overline{110}+40 \overline{100}-32$ \\
\hline & 5 & 6 & 1 & 2 & $\overline{400}+4 \overline{210}+4 \overline{211}-8 \overline{200}-6 \overline{110}+4 \overline{110}+4$ \\
\hline 28 & 5 & 7 & 1 & 2 & $\overline{400}+2 \overline{210}-4 \overline{200}-2 \overline{110}+4$ \\
\hline & 5 & 6 & 1 & 2 & $P(\mathscr{H}) P(\bullet)$ \\
\hline$\$$ & 5 & 7 & 1 & 6 & $P(\mathbb{\&}) P(\bullet)$ \\
\hline & 5 & 8 & 1 & 4 & $\overline{400}+\overline{210}-2 \overline{200}-2 \overline{100}+4$ \\
\hline \& & 5 & 7 & 1 & 4 & $\overline{400}+\overline{210}-2 \overline{200}+4 \overline{110}-10 \overline{100}+16$ \\
\hline & 5 & 8 & 1 & 8 & $\overline{400}+4 \overline{110}-8 \overline{100}+10$ \\
\hline$\$$ & 5 & 9 & 1 & 12 & $\overline{400}+2 \overline{110}-4 \overline{100}+4$ \\
\hline$K_{5}$ & 5 & 10 & 1 & 120 & $\overline{400}-2$ \\
\hline & 6 & 4 & 2 & 12 & $\overline{111} P(\bullet \bullet)^{4}$ \\
\hline & 6 & 4 & 2 & 8 & $\overline{111} P(\bullet \bullet)^{4}$ \\
\hline & 6 & 4 & 2 & 4 & $\overline{111} P(\bullet \bullet)^{4}$ \\
\hline 880 & 6 & 5 & 2 & 4 & $\overline{111} P(\boldsymbol{A}) P(\bullet \bullet)^{2}$ \\
\hline & 6 & 5 & 2 & 16 & $\overline{111} P(\boldsymbol{\bullet}) P(\bullet \bullet)^{2}$ \\
\hline$\triangle 8$ & 6 & 5 & 2 & 12 & $\overline{111} P(\boldsymbol{\diamond}) P(\bullet)^{2}$ \\
\hline & 6 & 6 & 2 & 8 & $\overline{111} P(\mathscr{2 0}) P(\bullet)$ \\
\hline & 6 & 6 & 2 & 72 & $\overline{111} P(\mathbf{d} \mathbf{6})^{2}$ \\
\hline$\$ 80^{\circ}$ & 6 & 7 & 2 & 48 & $\overline{111} P(\boldsymbol{\&}) P(\bullet \bullet)$ \\
\hline & 7 & 4 & 3 & 16 & $\overline{222} P($ ○) $) P(\bullet \bullet)^{2}$ \\
\hline AB & 7 & 5 & 3 & 48 & $\overline{222} P(\boldsymbol{\triangleleft}) P(\bullet)^{2}$ \\
\hline & 8 & 3 & 4 & 384 & $\overline{333} P(\bullet \bullet)^{4}$ \\
\hline
\end{tabular}

Table 3: The polynomial $P(G)$ for $v$-vertex graphs $G$ with $v-c(G)=4$. 


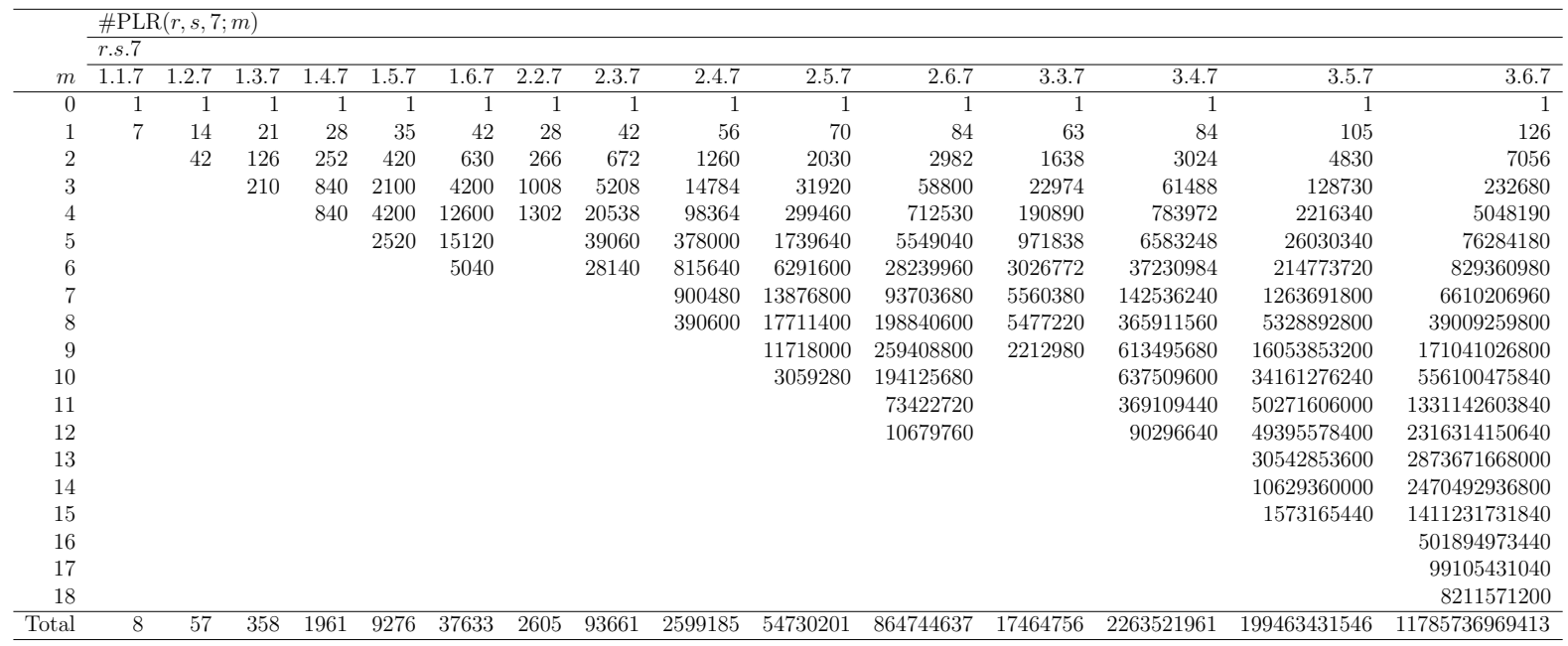

Table 4: The values of \#PLR $(r, s, 7 ; m)$; continued in Tables 5 and 6 .

\begin{tabular}{|c|c|c|c|c|c|c|}
\hline \multirow[b]{3}{*}{$m$} & \multicolumn{6}{|l|}{ \#PLR $(r, s, 7 ; m)$} \\
\hline & r.s.7 & & & & & \\
\hline & 4.4 .7 & 4.5 .7 & 4.6 .7 & 5.5 .7 & 5.6 .7 & 6.6 .7 \\
\hline 0 & 1 & 1 & 1 & 1 & 1 & 1 \\
\hline 1 & 112 & 140 & 168 & 175 & 210 & 252 \\
\hline 2 & 5544 & 8820 & 12852 & 14000 & 20370 & 29610 \\
\hline 3 & 160608 & 331800 & 594720 & 680400 & 1213800 & 2158800 \\
\hline 4 & 3040464 & 8342040 & 18654300 & 22520400 & 49851900 & 109648350 \\
\hline 5 & 39789792 & 148690080 & 421288560 & 539486640 & 1501095960 & 4129786080 \\
\hline 6 & 371511504 & 1945492080 & 7103917800 & 9705007200 & 34417437600 & 119886474960 \\
\hline 7 & 2518935552 & 19094265120 & 91553898240 & 134286297600 & 616139899200 & 2752801934400 \\
\hline 8 & 12508115256 & 142468484760 & 915820562160 & 1452407800200 & 8762762710800 & 50916808769400 \\
\hline 9 & 45551970240 & 814365132000 & 7182549494400 & 12413692800600 & 100228554703200 & 768744893767200 \\
\hline 10 & 121055555040 & 3578117047680 & 44440902031680 & 84446936458080 & 930070756954080 & 9567352024458480 \\
\hline 11 & 231977692800 & 12080294553600 & 217628184896640 & 459215324652000 & 7044643080720000 & 98867148338165760 \\
\hline 12 & 313967041920 & 31220730777600 & 844055906319360 & 2000199369924000 & 43729884582552000 & 852898268432422800 \\
\hline 13 & 290077079040 & 61311770150400 & 2588575554835200 & 6978471536484000 & 223002930233664000 & 6166083869012592000 \\
\hline 14 & 172656368640 & 90439590528000 & 6253755470524800 & 19466170012296000 & 935144001957312000 & 37457508269996136000 \\
\hline 15 & 59253304320 & 98519956738560 & 11829864008309760 & 43255879780478400 & 3223730533876070400 & 191508486724243180800 \\
\hline 16 & 8859553920 & 77323490294400 & 17371923533959680 & 76143045893544000 & 9122624741349504000 & 824650379018257377600 \\
\hline 17 & & 42126214233600 & 19575543305041920 & 105358455643896000 & 21136648322563200000 & 2990529904515892704000 \\
\hline 18 & & 14995766822400 & 16668621405273600 & 113411691586368000 & 39946627806672384000 & 9125647379336687472000 \\
\hline 19 & & 3114811929600 & 10507596032102400 & 93673159102656000 & 61276248720916992000 & 23396431711383803520000 \\
\hline 20 & & 284634362880 & 4768455577697280 & 58277161295539200 & 75809634470217446400 & 50284894217671092470400 \\
\hline 21 & & & 1496705022167040 & 26615217299328000 & 75050036025947136000 & 90334790420061996748800 \\
\hline 22 & & & 305223851842560 & 8591796855936000 & 58874808204632448000 & 135149758456395303936000 \\
\hline 23 & & & 36075091046400 & 1844984711808000 & 36156622400801280000 & 167647764880657152000000 \\
\hline 24 & & & 1862525145600 & 235436435136000 & 17119090026206784000 & 171521836534811629440000 \\
\hline 25 & & & & 13481774369280 & 6126452955671086080 & 143844493310595330785280 \\
\hline 26 & & & & & 1613475264781900800 & 98168168535490134466560 \\
\hline 27 & & & & & 300888959183769600 & 54057999485833839820800 \\
\hline 28 & & & & & 37371505393152000 & 23779699801418663424000 \\
\hline 29 & & & & & 2759601374208000 & 8256726182294360064000 \\
\hline 30 & & & & & 91288879718400 & 2230046357199562137600 \\
\hline 31 & & & & & & 459939642510304051200 \\
\hline 32 & & & & & & 70680227381503488000 \\
\hline 33 & & & & & & 7813153251735552000 \\
\hline 34 & & & & & & 587441307350016000 \\
\hline 35 & & & & & & 27048481121894400 \\
\hline 36 & & & & & & 583662346444800 \\
\hline Total & 1258840124753 & 435973408185561 & 92518523839617121 & 556422824213480176 & 407007072002505214801 & 982388579887448747338333 \\
\hline
\end{tabular}

Table 5: The values of \#PLR $(r, s, 7 ; m)$; continuing from Table 4 and continued in Table 6 . 


\begin{tabular}{|c|c|c|c|c|c|c|c|}
\hline \multirow[b]{3}{*}{$m$} & \multicolumn{7}{|c|}{ \#PLR $(r, 7,7 ; m)$} \\
\hline & $\begin{aligned} r .7 .7 \\
\end{aligned}$ & & & & & & \\
\hline & 1.7 .7 & 2.7 .7 & 3.7 .7 & 4.7 .7 & 5.7 .7 & 6.7 .7 & 7.7 .7 \\
\hline 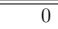 & 18 & 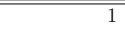 & 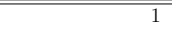 & $\begin{array}{ll}1 \\
\end{array}$ & 1 & 1 & 1 \\
\hline 1 & 49 & 98 & 147 & 196 & 245 & 294 & 343 \\
\hline 2 & 882 & 4116 & 9702 & 17640 & 27930 & 40572 & 55566 \\
\hline 3 & 7350 & 97608 & 381318 & 969024 & 1971270 & 3498600 & 5661558 \\
\hline 4 & 29400 & 1450302 & 9983358 & 36434244 & 96693660 & 211737330 & 407626002 \\
\hline 5 & 52920 & 14173740 & 184571730 & 996695280 & 3508057980 & 9577064700 & 22091837670 \\
\hline 6 & 35280 & 93118620 & 2493017100 & 20589037560 & 97824178200 & 336641627700 & 937499611860 \\
\hline 7 & 5040 & 413327040 & 25114127220 & 329058167760 & 2151220104600 & 9441643402800 & 31995541817820 \\
\hline 8 & & 1229208120 & 191003176980 & 4136301605520 & 37983532771800 & 215279839870200 & 895147467758460 \\
\hline 9 & & 2396605680 & 1103575119780 & 41356003473120 & 545522619369000 & 4045906316281200 & 20823534145010940 \\
\hline 10 & & 2949266880 & 4851540242640 & 331382137961280 & 6433667771868960 & 63326098060263360 & 407161408673448240 \\
\hline 11 & & 2154479040 & 16187551364880 & 2138171372830080 & 62740410283404000 & 832012118607983040 & 6747928605026748720 \\
\hline 12 & & 845696880 & 40729136096880 & 11136703296096000 & 508448168895240000 & 9231573671794519920 & 95414556472688784240 \\
\hline 13 & & 149516640 & 76460194354320 & 46850306414526720 & 3435977823932808000 & 86898548210325012000 & 1157011091919371520720 \\
\hline 14 & & 9344160 & 105451973716320 & 158998861707477120 & 19404150304485744000 & 696350429909011332000 & 12080739048610887859680 \\
\hline 15 & & & 104574912049440 & 434071037204501760 & 91672146486194601600 & 4762047565503866736000 & 108953982522887641120800 \\
\hline 16 & & & 72399498706080 & 948939730997852160 & 362335335766117560000 & 27837450938084937912000 & 850783267970671119386400 \\
\hline 17 & & & 33593118763680 & 1650710438532288000 & 1197163519923384216000 & 139234645196548772976000 & 5762016601975755442288800 \\
\hline 18 & & & 9863841496320 & 2265820889356362240 & 3300872875576140816000 & 596053661819256139968000 & 33886035458720657756006400 \\
\hline 19 & & & 1690904920320 & 2427993422686218240 & 7575781755486572208000 & 2183386963441728494016000 & 173162378870925255394329600 \\
\hline 20 & & & 151342732800 & 2003724888642247680 & 14423117979384567129600 & 6838094015249132148105600 & 769084553432477123576582400 \\
\hline 21 & & & 5411750400 & 1251798397787105280 & 22679370383067115200000 & 18286225276118547108614400 & 2968275252277138334102611200 \\
\hline 22 & & & & 579227971312972800 & 29296238052876891264000 & 41677467136757620221715200 & 9949401025065781152960038400 \\
\hline 23 & & & & 193034752263198720 & 30887319756977889408000 & 80769545715156012215424000 & 28936380037160064863828851200 \\
\hline 24 & & & & 44693646858846720 & 26372495140248144384000 & 132713702198233518483072000 & 72925724928137854106413900800 \\
\hline 25 & & & & 6860934701107200 & 18066619214217787207680 & 184255530135693426746542080 & 158995953693483311073403284480 \\
\hline 26 & & & & 655091210188800 & 9820001352274125465600 & 215290252940027718278891520 & 299284107563352671166958064640 \\
\hline 27 & & & & 34832706048000 & 4178611218476036966400 & 210725753874837580720373760 & 485219498022119678183816647680 \\
\hline 28 & & & & 782137036800 & 1369620935962581657600 & 171867215661546141628416000 & 675695410918704050010696483840 \\
\hline 29 & & & & & 339042043904814028800 & 116095014971808867619430400 & 805661431870384140528231336960 \\
\hline 30 & & & & & 61869133685050675200 & 64501823805957475751116800 & 819586476449488450769559091200 \\
\hline 31 & & & & & 8073669012853248000 & 29244277165012229350195200 & 708500236904008865987686041600 \\
\hline 32 & & & & & 723625308177408000 & 10722854433456169179033600 & 518142986889857917315006003200 \\
\hline 33 & & & & & 41905003262976000 & 3147095680488347470848000 & 318986018230847365565350041600 \\
\hline 34 & & & & & 1401648095232000 & 730644033010729291776000 & 164410170607527803740951142400 \\
\hline 35 & & & & & 20449013760000 & 132359825819298115584000 & 70519394490467460516228096000 \\
\hline 36 & & & & & & 18410847956245011456000 & 25006983924959260345820160000 \\
\hline 37 & & & & & & 1928283614905632768000 & 7279610083869038651882496000 \\
\hline 38 & & & & & & 148277402084431872000 & 1726520284027400861325312000 \\
\hline 39 & & & & & & 8074157388079104000 & 331008922550326911141888000 \\
\hline 40 & & & & & & 293524209893376000 & 50895029497370545118822400 \\
\hline 41 & & & & & & 6359357620224000 & 6228531360821639220019200 \\
\hline 42 & & & & & & 61479419904000 & 602622105599612348006400 \\
\hline 43 & & & & & & & 45850824283578118963200 \\
\hline 44 & & & & & & & 2734157863261981900800 \\
\hline 45 & & & & & & & 127631489644560384000 \\
\hline 46 & & & & & & & 4668091942993920000 \\
\hline 47 & & & & & & & 134218380312576000 \\
\hline 48 & & & & & & & 3012491575296000 \\
\hline 49 & & & & & & & 61479419904000 \\
\hline Total & 130922 & 10256288925 & 467281806581416 & 12027068084311265945 & 170054389801868987652126 & 1289970420801370588662084277 & 5175166233060627523665748739420 \\
\hline
\end{tabular}

Table 6: The values of $\# \operatorname{PLR}(r, 7,7 ; m)$. 


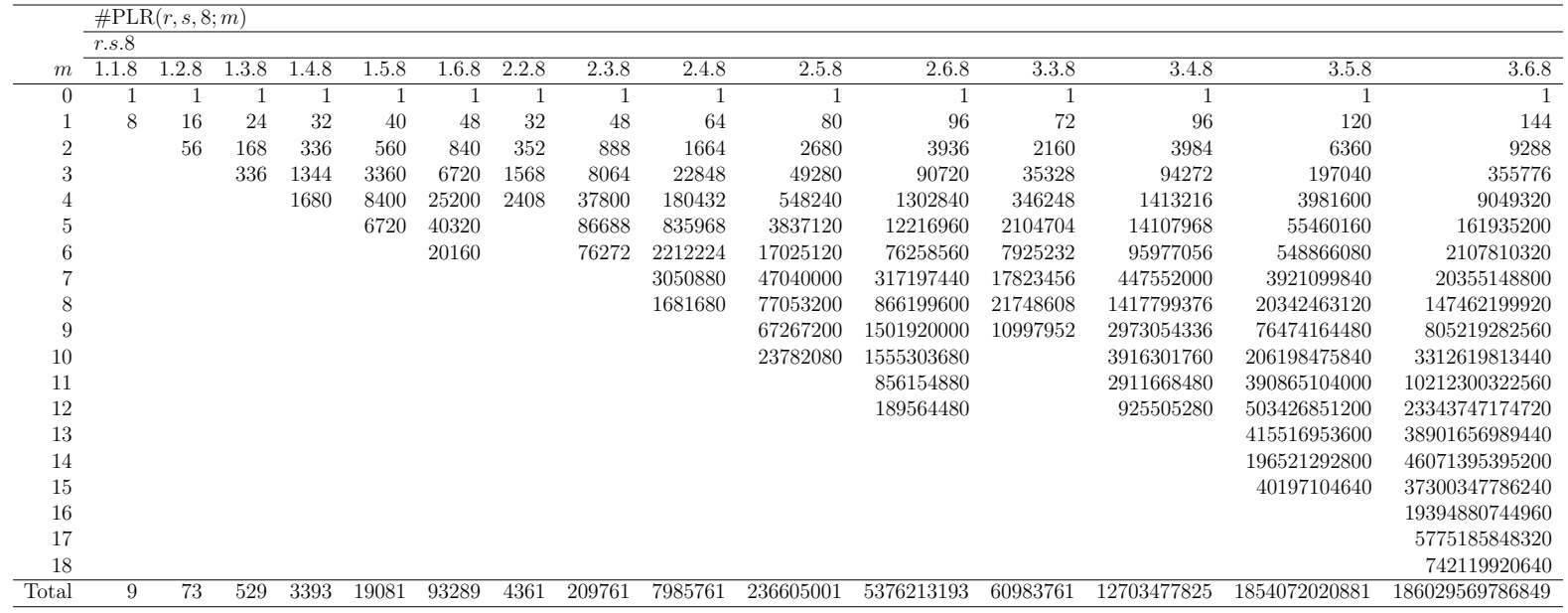

Table 7: The values of \#PLR $(r, s, 8 ; m)$; continued in Table 8.

\begin{tabular}{|c|c|c|c|c|c|c|}
\hline \multirow[b]{3}{*}{$m$} & \multicolumn{6}{|l|}{$\# \operatorname{PLR}(r, s, 8 ; m)$} \\
\hline & r.s.8 & & & & & \\
\hline & 4.4 .8 & 4.5 .8 & 4.6 .8 & 5.5 .8 & 5.6 .8 & 6.6 .8 \\
\hline 0 & 1 & 1 & 1 & 1 & 1 & 1 \\
\hline 1 & 128 & 160 & 192 & 200 & 240 & 288 \\
\hline 2 & 7296 & 11600 & 16896 & 18400 & 26760 & 38880 \\
\hline 3 & 245376 & 505920 & 905664 & 1035200 & 1844160 & 3275040 \\
\hline 4 & 5440032 & 14863920 & 33150960 & 39944000 & 88164000 & 193314600 \\
\hline 5 & 84155904 & 312224640 & 880629120 & 1123820160 & 3111238080 & 8513683200 \\
\hline 6 & 938106624 & 4857854400 & 17614343040 & 23931230400 & 84205144800 & 290863660800 \\
\hline 7 & 7674293760 & 57240046080 & 271706198400 & 395240496000 & 1792941696000 & 7913434233600 \\
\hline 8 & 46492403328 & 517971847680 & 3284306156880 & 5147427465600 & 30572202805200 & 174666634178400 \\
\hline 9 & 208994118144 & 3629706339840 & 31445326617600 & 53468641900800 & 422804060918400 & 3170532224025600 \\
\hline 10 & 693958185984 & 19775273602560 & 240125846929920 & 446402183619840 & 4786206531503040 & 47813256027210240 \\
\hline 11 & 1682575630848 & 83790652431360 & 1468289383142400 & 3010123202150400 & 44641974866227200 & 603661256780037120 \\
\hline 12 & 2918423765376 & 275253395880960 & 7199937958106880 & 16435824668659200 & 344653334210505600 & 6417774203497977600 \\
\hline 13 & 3499852769280 & 696318889996800 & 28293978724945920 & 72712045906752000 & 2208972817416960000 & 57702985271076096000 \\
\hline 14 & 2737429309440 & 1341720153849600 & 88858361994393600 & 260310345154272000 & 11772041299608844800 & 440141451429993062400 \\
\hline 15 & 1248707174400 & 1937078037135360 & 221915691372533760 & 751830550246218240 & 52182303997547888640 & 2854234197294902231040 \\
\hline 16 & 250631700480 & 2044882328832000 & 437480320642485120 & 1743112037427264000 & 192252531989997360000 & 15755740879827344094720 \\
\hline 17 & & 1520755813785600 & 673828360242831360 & 3220935488443008000 & 587611818336920832000 & 74072163230186875084800 \\
\hline 18 & & 749652906240000 & 799632015238103040 & 4696696282529664000 & 1485550685585627136000 & 296515269372479241369600 \\
\hline 19 & & 218552140800000 & 717288915035750400 & 5332415365638144000 & 3093542054560661760000 & 1009795856630569892352000 \\
\hline 20 & & 28375521914880 & 473551206050119680 & 4627930686056294400 & 5277311702476213478400 & 2921119683107942455372800 \\
\hline 21 & & & 221248992118210560 & 2991698111646720000 & 7323172199654814720000 & 7162134609634048893542400 \\
\hline 22 & & & 68717472783482880 & 1386151005947904000 & 8192740332265767936000 & 14840974457028794640384000 \\
\hline 23 & & & 12643342010449920 & 432666484604928000 & 7305506991725193216000 & 25896289714957972638720000 \\
\hline 24 & & & 1036744153804800 & 81104713998336000 & 5116406448359066112000 & 37882633011208622775936000 \\
\hline 25 & & & & & 2759923954130172641280 & 46209895877069315283271680 \\
\hline 26 & & & & & 1116269083866463027200 & 46698712018213236924579840 \\
\hline 27 & & & & & 325482156403465420800 & 38792083589752166760038400 \\
\hline 28 & & & & & 64264706091590860800 & 26235781041173371579699200 \\
\hline 29 & & & & & 7655207985266688000 & 14276150397241050415104000 \\
\hline 30 & & & & & 413733776530636800 & 6157077288600135234355200 \\
\hline 31 & & & & & & 2063745518966035159449600 \\
\hline 32 & & & & & & 523353569391869239296000 \\
\hline 33 & & & & & & 96567369329870143488000 \\
\hline 34 & & & & & & 12182875723557568512000 \\
\hline 35 & & & & & & 937008615326102323200 \\
\hline 36 & & & & & & 33087582858697113600 \\
\hline & 13295767306401 & 8920365218163361 & 3753438773423308993 & 25624385022295308521 & 42914661462094545592201 & 271169169298945362007111849 \\
\hline
\end{tabular}

Table 8: The values of \#PLR $(r, s, 8 ; m)$; continued from Table 7 . The present authors did not compute \#PLR $(r, 7,8 ; m)$ nor \#PLR $(r, 8,8 ; m)$. 


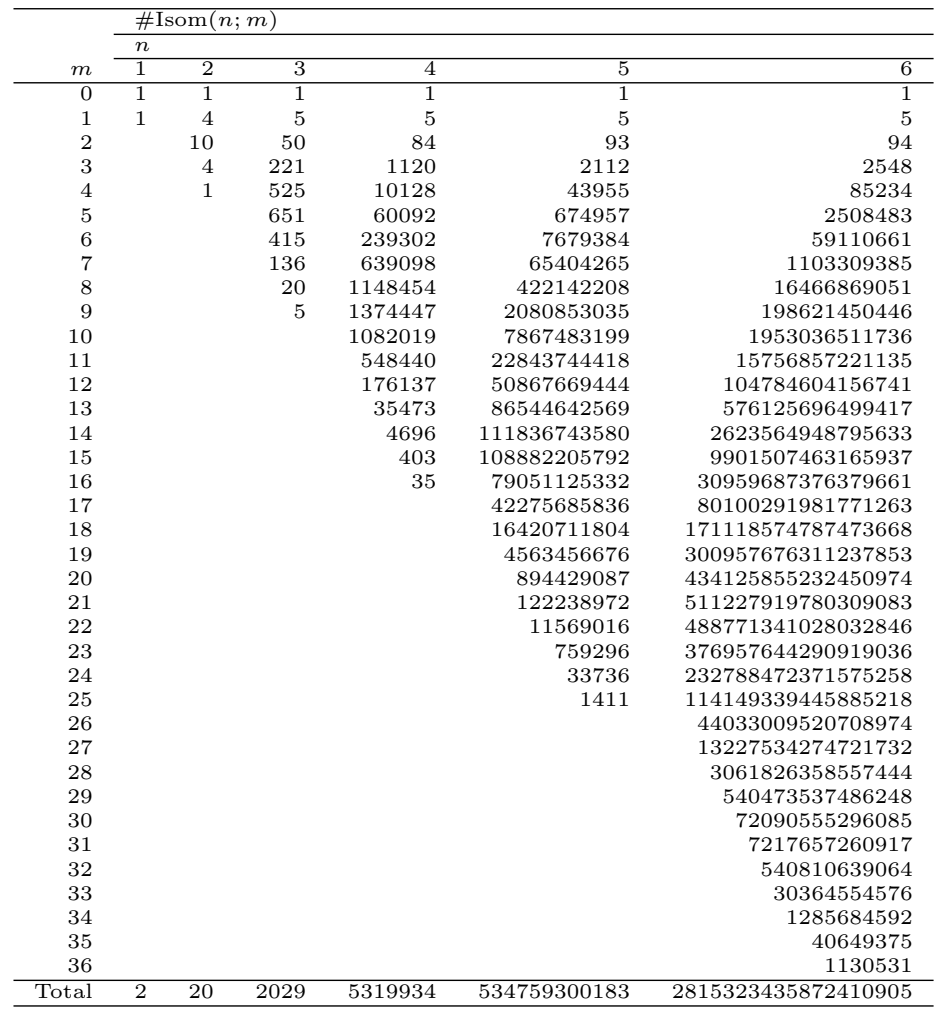

Table 9: Number of isomorphism classes in $\operatorname{PLS}(n ; m)$ for $n \leqslant 6$, according to weight $m$.

\begin{tabular}{|c|c|c|c|c|c|c|c|c|c|c|c|c|c|c|c|}
\hline \multirow[b]{3}{*}{$m$} & \multicolumn{15}{|c|}{$\# \operatorname{Isot}(2, s, n ; m)$} \\
\hline & \multicolumn{15}{|c|}{ 2.s.n } \\
\hline & 2.2 .2 & 2.2 .3 & 2.2 .4 & 2.2 .5 & 2.2 .6 & 2.3 .3 & 2.3 .4 & 2.3 .5 & 2.3 .6 & 2.4 .4 & 2.4 .5 & 2.4 .6 & 2.5 .5 & 2.5 .6 & 2.6 .6 \\
\hline 0 & 1 & 1 & 1 & 1 & 1 & 1 & 1 & 1 & 1 & 1 & 1 & 1 & 1 & 1 & 1 \\
\hline 1 & 1 & 1 & 1 & 1 & 1 & 1 & 1 & 1 & 1 & 1 & 1 & 1 & 1 & 1 & 1 \\
\hline 2 & 4 & 4 & 4 & 4 & 4 & 4 & 4 & 4 & 4 & 4 & 4 & 4 & 4 & 4 & 4 \\
\hline 3 & 1 & 2 & 2 & 2 & 2 & 5 & 5 & 5 & 5 & 5 & 5 & 5 & 5 & 5 & 5 \\
\hline 4 & 1 & 2 & 3 & 3 & 3 & 6 & 9 & 9 & 9 & 15 & 15 & 15 & 15 & 15 & 15 \\
\hline 5 & & & & & & 2 & 4 & 5 & 5 & 10 & 13 & 13 & 19 & 19 & 19 \\
\hline 6 & & & & & & 1 & 3 & 4 & 5 & 13 & 19 & 22 & 31 & 37 & 47 \\
\hline 7 & & & & & & & & & & 3 & 7 & 9 & 22 & 30 & 45 \\
\hline 8 & & & & & & & & & & 2 & 5 & 8 & 20 & 35 & 69 \\
\hline 9 & & & & & & & & & & & & & 5 & 12 & 40 \\
\hline 10 & & & & & & & & & & & & & 2 & 7 & 35 \\
\hline 11 & & & & & & & & & & & & & & & 7 \\
\hline 12 & & & & & & & & & & & & & & & 4 \\
\hline Total & 8 & 10 & 11 & 11 & 11 & 20 & 27 & 29 & 30 & 54 & 70 & 78 & 125 & 166 & 292 \\
\hline
\end{tabular}

Table 10: Number of isotopism classes in $\operatorname{PLR}(2, s, n ; m)$ for $2 \leqslant s \leqslant n \leqslant 6$, according to weight $m$. 


\begin{tabular}{|c|c|c|c|c|c|c|c|c|c|c|}
\hline \multirow[b]{3}{*}{$m$} & \multicolumn{10}{|c|}{$\# \operatorname{Isot}(3, s, n ; m)$} \\
\hline & \multicolumn{10}{|c|}{$3 . s . n$} \\
\hline & 3.3 .3 & 3.3 .4 & 3.3 .5 & 3.3 .6 & 3.4 .4 & 3.4 .5 & 3.4 .6 & 3.5 .5 & 3.5 .6 & 3.6 .6 \\
\hline 0 & 1 & 1 & 1 & 1 & 1 & 1 & 1 & 1 & 1 & 1 \\
\hline 1 & 1 & 1 & 1 & 1 & 1 & 1 & 1 & 1 & 1 & 1 \\
\hline 2 & 4 & 4 & 4 & 4 & 4 & 4 & 4 & 4 & 4 & 4 \\
\hline 3 & 11 & 11 & 11 & 11 & 11 & 11 & 11 & 11 & 11 & 11 \\
\hline 4 & 18 & 25 & 25 & 25 & 36 & 36 & 36 & 36 & 36 & 36 \\
\hline 5 & 23 & 42 & 49 & 49 & 78 & 91 & 91 & 109 & 109 & 109 \\
\hline 6 & 15 & 52 & 71 & 77 & 174 & 237 & 254 & 330 & 356 & 389 \\
\hline 7 & 6 & 33 & 70 & 82 & 215 & 430 & 502 & 858 & 1012 & 1212 \\
\hline 8 & 1 & 11 & 34 & 52 & 192 & 585 & 855 & 1770 & 2568 & 3782 \\
\hline 9 & 1 & 4 & 13 & 23 & 91 & 491 & 962 & 2683 & 5168 & 10001 \\
\hline 10 & & & & & 30 & 257 & 740 & 2689 & 7706 & 21857 \\
\hline 11 & & & & & 4 & 71 & 298 & 1794 & 7988 & 35822 \\
\hline 12 & & & & & 2 & 12 & 70 & 709 & 5446 & 42768 \\
\hline 13 & & & & & & & & 177 & 2301 & 34916 \\
\hline 14 & & & & & & & & 19 & 530 & 19078 \\
\hline 15 & & & & & & & & 3 & 62 & 6441 \\
\hline 16 & & & & & & & & & & 1315 \\
\hline 17 & & & & & & & & & & 133 \\
\hline 18 & & & & & & & & & & 16 \\
\hline Total & 81 & 184 & 279 & 325 & 839 & 2227 & 3825 & 11194 & 33299 & 177892 \\
\hline
\end{tabular}

Table 11: Number of isotopism classes in $\operatorname{PLR}(3, s, n ; m)$ for $3 \leqslant s \leqslant n \leqslant 6$, according to weight $m$. 


\begin{tabular}{|c|c|c|c|c|c|c|c|c|c|c|}
\hline \multirow[b]{3}{*}{$m$} & \multicolumn{10}{|c|}{$\# \operatorname{Isot}(r, s, n ; m)$} \\
\hline & r.s.n & & & & & & & & & \\
\hline & 4.4 .4 & 4.4 .5 & 4.4 .6 & 4.5 .5 & 4.5 .6 & 4.6 .6 & 5.5 .5 & 5.5 .6 & 5.6 .6 & 6.6 .6 \\
\hline 0 & 1 & 1 & 1 & 1 & 1 & 1 & 1 & 1 & 1 & 1 \\
\hline 1 & 1 & 1 & 1 & 1 & 1 & 1 & 1 & 1 & 1 & 1 \\
\hline 2 & 4 & 4 & 4 & 4 & 4 & 4 & 4 & 4 & 4 & 4 \\
\hline 3 & 11 & 11 & 11 & 11 & 11 & 11 & 11 & 11 & 11 & 11 \\
\hline 4 & 52 & 52 & 52 & 52 & 52 & 52 & 52 & 52 & 52 & 52 \\
\hline 5 & 139 & 160 & 160 & 187 & 187 & 187 & 221 & 221 & 221 & 221 \\
\hline 6 & 507 & 668 & 707 & 882 & 935 & 997 & 1158 & 1227 & 1306 & 1396 \\
\hline 7 & 1161 & 2103 & 2395 & 3713 & 4223 & 4826 & 6310 & 7127 & 8064 & 9130 \\
\hline 8 & 2136 & 5678 & 7754 & 14266 & 19080 & 25524 & 33293 & 43322 & 56110 & 72145 \\
\hline 9 & 2429 & 10739 & 19067 & 42940 & 72764 & 121508 & 150964 & 241958 & 380083 & 583339 \\
\hline 10 & 2004 & 14881 & 36957 & 99301 & 230072 & 515040 & 554285 & 1174047 & 2384388 & 4627607 \\
\hline 11 & 975 & 13865 & 50826 & 168900 & 565202 & 1797295 & 1594532 & 4699600 & 12974453 & 33362634 \\
\hline 12 & 364 & 8970 & 50244 & 210285 & 1064946 & 5054807 & 3539461 & 15159299 & 59361654 & 210409407 \\
\hline 13 & 72 & 3664 & 32727 & 187214 & 1498530 & 11135187 & 6017824 & 38833501 & 223569607 & 1129335392 \\
\hline 14 & 18 & 995 & 13973 & 117985 & 1557518 & 19016101 & 7772366 & 78368607 & 686354327 & 5091624997 \\
\hline 15 & 2 & 141 & 3268 & 51094 & 1166309 & 24794117 & 7568187 & 123670028 & 1706058231 & 19140028219 \\
\hline 16 & 2 & 22 & 411 & 14960 & 616603 & 24415585 & 5493206 & 151457082 & 3417379856 & 59761963636 \\
\hline 17 & & & & 2814 & 220158 & 17834146 & 2939617 & 142614087 & 5488132262 & 154544375137 \\
\hline 18 & & & & 332 & 50723 & 9492300 & 1141472 & 102078688 & 7025903964 & 330108625102 \\
\hline 19 & & & & 24 & 6591 & 3575605 & 317980 & 54746803 & 7119415871 & 580559388329 \\
\hline 20 & & & & 3 & 428 & 926317 & 62319 & 21601198 & 5662138638 & 837440466326 \\
\hline 21 & & & & & & 156463 & 8676 & 6121385 & 3498117999 & 986167409118 \\
\hline 22 & & & & & & 16759 & 823 & 1203460 & 1658503251 & 942850011453 \\
\hline 23 & & & & & & 960 & 69 & 155952 & 594594494 & 727157075193 \\
\hline 24 & & & & & & 56 & 6 & 12023 & 158425032 & 449054224783 \\
\hline 25 & & & & & & & 2 & 486 & 30703736 & 220195944263 \\
\hline 26 & & & & & & & & & 4220807 & 84941236104 \\
\hline 27 & & & & & & & & & 396518 & 25516234965 \\
\hline 28 & & & & & & & & & 24531 & 5906586539 \\
\hline 29 & & & & & & & & & 886 & 1042616896 \\
\hline 30 & & & & & & & & & 40 & 139114631 \\
\hline 31 & & & & & & & & & & 13928529 \\
\hline 32 & & & & & & & & & & 1048656 \\
\hline 33 & & & & & & & & & & 59130 \\
\hline 34 & & & & & & & & & & 2846 \\
\hline 35 & & & & & & & & & & 109 \\
\hline 36 & & & & & & & & & & 22 \\
\hline Total & 9878 & 61955 & 218558 & 914969 & 7074338 & 118883849 & 37202840 & 742190170 & 37349106398 & 5431010366323 \\
\hline
\end{tabular}

Table 12: Number of isotopism classes in $\operatorname{PLR}(r, s, n ; m)$ for $4 \leqslant r \leqslant s \leqslant n \leqslant 6$, according to weight $m$. 


\begin{tabular}{|c|c|c|c|c|c|c|c|c|c|c|c|c|c|c|c|c|}
\hline \multirow[b]{3}{*}{$m$} & \multicolumn{16}{|c|}{$\# \mathrm{MC}(r, s, n ; m)$} \\
\hline & \multicolumn{16}{|c|}{ r.s.n } \\
\hline & 2.2 .2 & 2.2 .3 & 2.2 .4 & 2.2 .5 & 2.2 .6 & 2.3 .3 & 2.4 .4 & 2.5 .5 & 2.6 .6 & 3.3 .3 & 3.3 .4 & 3.3 .5 & 3.3 .6 & 3.4 .4 & 3.5 .5 & 3.6 .6 \\
\hline 0 & 1 & 1 & 1 & 1 & 1 & 1 & 1 & 1 & 1 & 1 & 1 & 1 & 1 & 1 & 1 & 1 \\
\hline 1 & 1 & 1 & 1 & 1 & 1 & 1 & 1 & 1 & 1 & 1 & 1 & 1 & 1 & 1 & 1 & 1 \\
\hline 2 & 2 & 3 & 3 & 3 & 3 & 3 & 3 & 3 & 3 & 2 & 3 & 3 & 3 & 3 & 3 & 3 \\
\hline 3 & 1 & 2 & 2 & 2 & 2 & 4 & 4 & 4 & 4 & 5 & 8 & 8 & 8 & 8 & 8 & 8 \\
\hline 4 & 1 & 2 & 3 & 3 & 3 & 5 & 11 & 11 & 11 & 8 & 18 & 18 & 18 & 24 & 24 & 24 \\
\hline 5 & & & & & & 2 & 8 & 14 & 14 & 9 & 28 & 33 & 33 & 49 & 67 & 67 \\
\hline 6 & & & & & & 1 & 10 & 22 & 32 & 7 & 34 & 46 & 50 & 104 & 191 & 224 \\
\hline 7 & & & & & & & 3 & 17 & 32 & 4 & 23 & 46 & 54 & 128 & 477 & 667 \\
\hline 8 & & & & & & & 2 & 16 & 48 & 1 & 9 & 24 & 36 & 116 & 963 & 2018 \\
\hline 9 & & & & & & & & 5 & 31 & 1 & 4 & 12 & 20 & 59 & 1444 & 5233 \\
\hline 10 & & & & & & & & 2 & 27 & & & & & 22 & 1452 & 11309 \\
\hline 11 & & & & & & & & & 7 & & & & & 4 & 986 & 18435 \\
\hline 12 & & & & & & & & & 4 & & & & & 2 & 411 & 21995 \\
\hline 13 & & & & & & & & & & & & & & & 115 & 18028 \\
\hline 14 & & & & & & & & & & & & & & & 16 & 9969 \\
\hline 15 & & & & & & & & & & & & & & & 3 & 3468 \\
\hline 16 & & & & & & & & & & & & & & & & 765 \\
\hline 17 & & & & & & & & & & & & & & & & 94 \\
\hline 18 & & & & & & & & & & & & & & & & 14 \\
\hline Total & 6 & 9 & 10 & 10 & 10 & 17 & 43 & 96 & 215 & 39 & 129 & 192 & 224 & 521 & 6162 & 92323 \\
\hline
\end{tabular}

Table 13: Number of main classes in $\operatorname{PLR}(r, s, n ; m)$ for $2 \leqslant r \leqslant s \leqslant n \leqslant 6$ with $r \leqslant 3$, according to weight $m$. We omit the cases when $r, s$, and $n$ are pairwise distinct. 


\begin{tabular}{|c|c|c|c|c|c|c|c|c|c|}
\hline \multirow[b]{3}{*}{$m$} & \multicolumn{9}{|c|}{ \#MC $(r, s, n ; m)$} \\
\hline & r.s.n & & & & & & & & \\
\hline & 4.4 .4 & 4.4 .5 & 4.4 .6 & 4.5 .5 & 4.6 .6 & 5.5 .5 & 5.5 .6 & 5.6 .6 & 6.6 .6 \\
\hline 0 & 1 & 1 & 1 & 1 & 1 & 1 & 1 & 1 & 1 \\
\hline 1 & 1 & 1 & 1 & 1 & 1 & 1 & 1 & 1 & 1 \\
\hline 2 & 2 & 3 & 3 & 3 & 3 & 2 & 3 & 3 & 2 \\
\hline 3 & 5 & 8 & 8 & 8 & 8 & 5 & 8 & 8 & 5 \\
\hline 4 & 18 & 34 & 34 & 34 & 34 & 18 & 34 & 34 & 18 \\
\hline 5 & 39 & 97 & 97 & 111 & 111 & 59 & 131 & 131 & 59 \\
\hline 6 & 121 & 376 & 399 & 489 & 551 & 256 & 677 & 717 & 306 \\
\hline 7 & 253 & 1135 & 1293 & 1969 & 2548 & 1224 & 3748 & 4225 & 1747 \\
\hline 8 & 442 & 2987 & 4070 & 7392 & 13142 & 5997 & 22209 & 28670 & 12799 \\
\hline 9 & 495 & 5579 & 9847 & 21936 & 61657 & 26188 & 122390 & 191855 & 99715 \\
\hline 10 & 420 & 7694 & 18939 & 50420 & 259637 & 94479 & 590423 & 1197283 & 779295 \\
\hline 11 & 218 & 7170 & 25943 & 85477 & 902847 & 269456 & 2356900 & 6500092 & 5583650 \\
\hline 12 & 96 & 4686 & 25682 & 106351 & 2535116 & 595649 & 7593131 & 29710547 & 35131875 \\
\hline 13 & 25 & 1944 & 16768 & 94754 & 5579487 & 1010706 & 19438925 & 111845936 & 188377998 \\
\hline 14 & 8 & 561 & 7283 & 59910 & 9524578 & 1304319 & 39216773 & 343290367 & 848955581 \\
\hline 15 & 2 & 88 & 1742 & 26146 & 12416456 & 1270356 & 61876720 & 853215848 & 3190714878 \\
\hline 16 & 2 & 19 & 262 & 7790 & 12227832 & 923128 & 75774954 & 1708965453 & 9961645532 \\
\hline 17 & & & & 1533 & 8934309 & 495565 & 71353021 & 2744426741 & 25759586139 \\
\hline 18 & & & & 200 & 4758913 & 193531 & 51076956 & 3513371841 & 55021427957 \\
\hline 19 & & & & 19 & 1795458 & 54746 & 27401728 & 3560137618 & 96764408110 \\
\hline 20 & & & & 3 & 467000 & 11052 & 10816787 & 2831458432 & 139578978645 \\
\hline 21 & & & & & 79692 & 1693 & 3069771 & 1749363542 & 164367335977 \\
\hline 22 & & & & & 8815 & 192 & 604997 & 829461470 & 157147744329 \\
\hline 23 & & & & & 566 & 26 & 79301 & 297418767 & 121198141862 \\
\hline 24 & & & & & 44 & 4 & 6249 & 79274342 & 74846573994 \\
\hline 25 & & & & & & 2 & 312 & 15377110 & 36702176578 \\
\hline 26 & & & & & & & & 2119455 & 14158650257 \\
\hline 27 & & & & & & & & 200664 & 4253618044 \\
\hline 28 & & & & & & & & 12830 & 984869538 \\
\hline 29 & & & & & & & & 527 & 173933415 \\
\hline 30 & & & & & & & & 33 & 23245431 \\
\hline 31 & & & & & & & & & 2336988 \\
\hline 32 & & & & & & & & & 179057 \\
\hline 33 & & & & & & & & & 10603 \\
\hline 34 & & & & & & & & & 640 \\
\hline 35 & & & & & & & & & 40 \\
\hline 36 & & & & & & & & & 12 \\
\hline Total & 2148 & 32383 & 112372 & 464547 & 59568806 & 6239377 & 371406150 & 18677574543 & 905214521078 \\
\hline
\end{tabular}

Table 14: Number of main classes in $\operatorname{PLR}(r, s, n ; m)$ for $4 \leqslant r \leqslant s \leqslant n \leqslant 6$, according to weight $m$. We omit the cases when $r, s$, and $n$ are pairwise distinct.

\begin{tabular}{rrr}
\hline$m$ & \#Isot $(r, s, n ; m)$ & $\# \mathrm{MC}(r, s, n ; m)$ \\
\hline 0 & 1 & 1 \\
1 & 1 & 1 \\
2 & 4 & 2 \\
3 & 11 & 5 \\
4 & 52 & 18 \\
5 & 221 & 59 \\
6 & 1396 & 306 \\
7 & 9719 & 1861 \\
8 & 85145 & 15097 \\
9 & 860347 & 146893 \\
10 & 10071270 & 1693416 \\
11 & 133048009 & 22239872 \\
12 & $?$ & 327670703 \\
\hline
\end{tabular}

Table 15: Number of isotopism classes and main classes in $\operatorname{PLR}(r, s, n ; m)$ when $r \geqslant m$, $s \geqslant m$, and $n \geqslant m$, according to weight $m$. The second column is Sloane's oeis.org/ A286317. 